\title{
Thermal right-handed neutrino production rate in the relativistic regime
}

\section{Laine}

Institute for Theoretical Physics, Albert Einstein Center, University of Bern, Sidlerstrasse 5, CH-3012 Bern, Switzerland

E-mail: laine@itp.unibe.ch

ABSTRACT: The production rate of right-handed neutrinos from a Standard Model plasma at a temperature above a hundred $\mathrm{GeV}$ is evaluated up to NLO in Standard Model couplings. The results apply in the so-called relativistic regime, referring parametrically to a mass $M \sim \pi T$, generalizing thereby previous NLO results which only apply in the nonrelativistic regime $M \gg \pi T$. The non-relativistic expansion is observed to converge for $M \gtrsim 15 T$, but the smallness of any loop corrections allows it to be used in practice already for $M \gtrsim 4 T$. In the latter regime any non-covariant dependence of the differential rate on the spatial momentum is shown to be mild. The loop expansion breaks down in the ultrarelativistic regime $M \ll \pi T$, but after a simple mass resummation it nevertheless extrapolates reasonably well towards a result obtained previously through complete LPM resummation, apparently confirming a strong enhancement of the rate at high temperatures (which facilitates chemical equilibration). When combined with other ingredients the results may help to improve upon the accuracy of leptogenesis computations operating above the electroweak scale.

Keywords: Thermal Field Theory, Neutrino Physics, Resummation

ARXIV EPRINT: 1307.4909 


\section{Contents}

1 Introduction 1

2 Setup 2

3 Main results 4

3.1 Strict NLO expression 4

$\begin{array}{lll}3.2 & \text { Non-relativistic limit } & 6\end{array}$

$\begin{array}{lll}3.3 & \text { Towards ultra-relativistic limit } & 6\end{array}$

4 Spectra and spectral functions $\quad 10$

$\begin{array}{llr}5 & \text { Total production rate } & 10\end{array}$

6 Conclusions and outlook 11

$\begin{array}{ll}\text { A Definitions of master sum-integrals } & 13\end{array}$

B Results for master spectral functions in time-like domain $\quad \mathbf{1 5}$

$\begin{array}{lll}\text { B.1 } \rho_{\mathcal{J}_{\mathrm{a}}} & 15\end{array}$

$\begin{array}{lll}\text { B.2 } & \rho_{\mathcal{J}_{\mathrm{b}}} & 15\end{array}$

$\begin{array}{lll}\text { B.3 } & \rho_{\mathcal{I}_{\mathrm{b}}} & 17\end{array}$

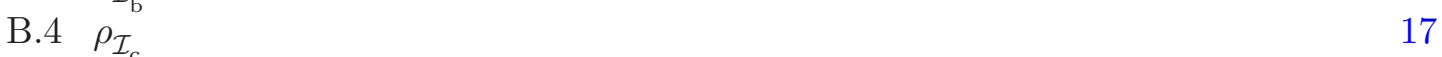

B.5 $\rho_{\mathcal{I}_{\mathrm{d}}} \quad 18$

$\begin{array}{llr}\text { B.6 } & \rho_{\mathcal{I}_{\mathrm{e}}} & 18\end{array}$

$\begin{array}{lll}\text { B.7 } & \rho_{\mathcal{I}_{\mathrm{f}}} & 19\end{array}$

$\begin{array}{lll}\text { B. } 8 & \rho_{\mathcal{I}_{\mathrm{g}}} & 21\end{array}$

B.9 $\rho_{\mathcal{I}_{\mathrm{h}}} \quad 22$

$\begin{array}{ll}\text { B.10 } \rho_{\mathcal{I}_{\mathrm{h}},} & 27\end{array}$

$\begin{array}{ll}\text { C Choice of parameters } & 31\end{array}$

\section{Introduction}

The neutrino sector is arguably the least precisely charted among the different parts of experimentally accessible particle physics. Whereas it is well established that at least two dominantly left-handed neutrinos have masses, and that the mass eigenstates are misaligned with the weak interaction eigenstates, already the absolute value of the mass scale remains poorly constrained. There is also feeble empirical handle on the dynamics of neutrino mass generation, although a see-saw mechanism involving Majorana masses of 
gauge-singlet right-handed neutrinos is a natural candidate. Of course right-handed neutrinos can be introduced in any case, but then a large parameter space of Yukawa couplings and Majorana masses, the latter unbounded from above, remains available to phenomenological consideration.

The big volume of the parameter space suggests seeking for cosmological constraints on neutrino properties. Apart from the well-studied significance of left-handed neutrinos to the overall expansion rate of the Universe through the pressure and energy density that they exert, it is also possible that right-handed neutrinos have cosmological significance. For instance, they could play a role in explaining two outstanding cosmological mysteries, the existence of a matter-antimatter asymmetry [1] (for reviews, see e.g. refs. [2, 3]) as well as the existence of particle dark matter [4] (for a review see e.g. ref. [5]).

The present paper is related to developing theoretical tools for studying right-handed neutrinos within a cosmological environment. It has been understood recently [6-8] that in the so-called ultrarelativistic regime $\pi T \gg M$, where $T$ denotes the temperature and $M$ a right-handed neutrino Majorana mass parameter, the thermal loop expansion breaks down and needs to be resummed with techniques analogous to those that were previously developed in the context of photon production from a hot QCD plasma [9-11]. In contrast, in the so-called non-relativistic regime $\pi T \ll M$, next-to-leading order (NLO) corrections can be computed and are in general small $[12,13]$, in accordance with expectations based on the Operator Product Expansion (OPE) [14]. This leaves open the question of how these two qualitatively very different regimes interpolate to each other.

The purpose of the present paper is to present a theoretically consistent computation of the right-handed neutrino production rate in the so-called relativistic regime, $\pi T \sim M$. The principal tools needed for this have been developed in ref. [15], and here we assemble the full results. We also inspect under which conditions the results go over to those of the limiting ultrarelativistic and non-relativistic cases. In addition the structure of the differential production rate is analyzed with the goal of suggesting a numerically affordable and yet relatively accurate approximation scheme that may be used in practical applications (however the study of practical applications is postponed to future work). Another project with partly similar goals has recently been outlined in ref. [16].

After summarizing the setup in section 2, the basic theoretical results, together with comparisons with the non-relativistic and ultrarelativistic regimes, are presented in section 3. Examples of numerical results for differential production spectra are shown in section 4 , whereas in section 5 the total production rate is considered. Some conclusions and an outlook are offered in section 6 .

\section{Setup}

Solving the Liouville-von Neumann equation for the time evolution of the density matrix of a coupled system of Standard Model particles and right-handed neutrinos to leading non-trivial order in neutrino Yukawa couplings (but to all orders in Standard Model couplings), for times sufficiently small that the right-handed neutrinos do not chemically 
equilibrate [17], it is found that their "differential production rate" can be written as

$$
\left.\frac{\mathrm{d} N_{+}(\mathcal{K})}{\mathrm{d}^{4} \mathcal{X} \mathrm{d}^{3} \mathbf{k}} \equiv \frac{\mathrm{d} N(\mathcal{K})}{\mathrm{d}^{4} \mathcal{X} \mathrm{d}^{3} \mathbf{k}}\right|_{\frac{\mathrm{d} N(\mathcal{K})}{\mathrm{d}^{3} \mathbf{x} \mathrm{d}^{3} \mathbf{k}} \approx 0}=\frac{2 n_{\mathrm{F}}\left(k_{0}\right)}{(2 \pi)^{3}} \Gamma(\mathcal{K})
$$

Here a "width" has been defined as

$$
\Gamma(\mathcal{K}) \equiv \frac{1}{k_{0}} \operatorname{Im} \Pi_{\mathrm{R}}(\mathcal{K})=\frac{1}{k_{0}} \operatorname{Im}\left\{\Pi_{\mathrm{E}}(K)\right\}_{k_{n} \rightarrow-i\left[k_{0}+i 0^{+}\right]},
$$

where $\Pi_{\mathrm{E}}$ is a gauge-invariant and Lorentz-singlet 2-point correlation function of the "currents" that right-handed neutrinos couple to,

$$
\Pi_{\mathrm{E}}(K) \equiv\left|h_{\nu \mathrm{B}}\right|^{2} \operatorname{Tr}\left\{i \not K\left[\int_{0}^{1 / T} \mathrm{~d} \tau \int_{\mathbf{x}} e^{i K \cdot X}\left\langle\left(\tilde{\phi}^{\dagger} a_{\mathrm{L}} \ell\right)(X)\left(\bar{\ell} a_{\mathrm{R}} \tilde{\phi}\right)(0)\right\rangle_{T}\right]\right\}
$$

and $\Pi_{R}$ is the corresponding retarded real-time correlator (its imaginary part equals the spectral function). Moreover, $h_{\nu \mathrm{B}}$ denotes a bare neutrino Yukawa coupling (or, more generally, elements of a Yukawa matrix); Euclidean variables are denoted by $X \equiv(\tau, \mathbf{x})$, $K \equiv\left(k_{n}, \mathbf{k}\right)$; the corresponding Minkowskian ones by $\mathcal{X} \equiv(t, \mathbf{x}), \mathcal{K} \equiv\left(k_{0}, \mathbf{k}\right)$; the metric conventions are $K^{2}=k_{n}^{2}+k^{2}, \mathcal{K}^{2}=k_{0}^{2}-k^{2}$, with $k \equiv|\mathbf{k}| ; k_{n}$ stands for a fermionic Matsubara frequency, reflecting the fact that spin- $\frac{1}{2}$ fields are antiperiodic across the Euclidean time direction of extent $1 / T$. (Definitions of other variables appearing in eq. (2.3) can be found in ref. [13].) A corresponding integrated "total production rate" is

$$
\gamma_{+}(T) \equiv \frac{\mathrm{d} N_{+}}{\mathrm{d}^{4} \mathcal{X}}=\int \frac{\mathrm{d}^{3} \mathbf{k}}{(2 \pi)^{3}} 2 n_{\mathrm{F}}\left(k_{0}\right) \Gamma(\mathcal{K})
$$

We note in passing that an opposite case of a "differential decay rate", defined by taking the thermal average of a spin-summed rate for the disappearance of a right-handed neutrino of momentum $\mathbf{k}$, can be expressed in terms of the same function $\Gamma(\mathcal{K})$ that appears in eq. (2.2):

$$
\frac{\mathrm{d} N_{-}(\mathcal{K})}{\mathrm{d}^{4} \mathcal{X} \mathrm{d}^{3} \mathbf{k}}=-\frac{2\left[1-n_{\mathrm{F}}\left(k_{0}\right)\right]}{(2 \pi)^{3}} \Gamma(\mathcal{K})
$$

The observables above are particularly simple because they involve a sum over the spin states of the right-handed neutrinos. A more general problem concerns the determination of the self-energy matrix of the right-handed neutrinos, given to leading order in neutrino Yukawa couplings by eq. (2.3) without a Dirac contraction with $i \not K$. An NLO discussion of this observable in the non-relativistic regime can be found in ref. [18].

Returning to eq. (2.3), one of the strengths of the imaginary-time formulation of thermal field theory is that the expression obtained for $\Pi_{\mathrm{E}}$ can be significantly simplified through substitutions of loop momenta before taking the cut leading to $\Gamma$. In fact, as shown in ref. [13], $\Pi_{\mathrm{E}}$ can be represented in terms of a small number of "master" sumintegrals. For the specific case of naive dimensional regularization of the $\gamma_{5}$-matrix, the 
expression reads

$$
\begin{aligned}
\frac{\Pi_{\mathrm{E}}}{\left|h_{\nu \mathrm{B}}\right|^{2}=} & 2\left(\widetilde{\mathcal{J}}_{\mathrm{a}}-\mathcal{J}_{\mathrm{a}}-\mathcal{J}_{\mathrm{b}}\right) \\
& +12 \lambda_{\mathrm{B}}\left(-\mathcal{I}_{\mathrm{b}}+\mathcal{I}_{\mathrm{c}}+\mathcal{I}_{\mathrm{d}}\right) \\
& +2 h_{t \mathrm{~B}}^{2} N_{\mathrm{c}}\left[2\left(\widetilde{\mathcal{I}}_{\mathrm{b}}-\widetilde{\mathcal{I}}_{\mathrm{c}}-\widetilde{\mathcal{I}}_{\mathrm{d}}\right)+\widetilde{\mathcal{I}}_{\mathrm{e}}-\widetilde{\mathcal{I}}_{\mathrm{f}}+\widetilde{\mathcal{I}}_{\mathrm{h}}\right] \\
& +\frac{g_{1 \mathrm{~B}}^{2}+3 g_{2 \mathrm{~B}}^{2}}{2}\left[-\mathcal{I}_{\mathrm{b}}+2\left(\widetilde{\mathcal{I}}_{\mathrm{e}}-\mathcal{I}_{\mathrm{e}}+\mathcal{I}_{\mathrm{g}}+\mathcal{I}_{\mathrm{j}}\right)-4\left(\mathcal{I}_{\mathrm{h}}+\widehat{\mathcal{I}}_{\mathrm{h}}\right)\right. \\
& \left.+(D-1)\left(\mathcal{I}_{\mathrm{c}}+\mathcal{I}_{\mathrm{d}}\right)+(D-2)\left(\overline{\mathcal{I}}_{\mathrm{c}}-\overline{\mathcal{I}}_{\mathrm{d}}-\widetilde{\mathcal{I}}_{\mathrm{b}}-\widehat{\mathcal{I}}_{\mathrm{c}}+\widehat{\mathcal{I}}_{\mathrm{d}}+\widehat{\mathcal{I}}_{\mathrm{h}}\right)\right],
\end{aligned}
$$

where $\lambda_{\mathrm{B}}, h_{t \mathrm{~B}}, g_{1 \mathrm{~B}}, g_{2 \mathrm{~B}}$ denote the bare Higgs, top Yukawa, U(1) gauge, and $\mathrm{SU}(2)$ gauge couplings, respectively; $N_{\mathrm{c}}=3$ is the number of colours; and $D \equiv 4-2 \epsilon$ is the space-time dimensionality. The definitions of the independent master sum-integrals $\mathcal{J}_{\mathrm{a}}, \ldots$ are listed in appendix A. Renormalization of this bare expression is achieved through

$$
\left|h_{\nu \mathrm{B}}\right|^{2}=\left|h_{\nu}(\bar{\mu})\right|^{2} \mu^{2 \epsilon} \mathcal{Z}_{\nu}, \quad \mathcal{Z}_{\nu} \equiv 1+\frac{1}{(4 \pi)^{2} \epsilon}\left[h_{t}^{2} N_{\mathrm{c}}-\frac{3}{4}\left(g_{1}^{2}+3 g_{2}^{2}\right)\right]
$$

where $\mu$ is a scale parameter related to dimensional regularization (in the following inconsequential factors $\mu^{ \pm 2 \epsilon}$ are omitted); the $\overline{\mathrm{MS}}$ scale is defined as $\bar{\mu}^{2} \equiv 4 \pi \mu^{2} e^{-\gamma_{\mathrm{E}}}$; and $h_{t}, g_{1}, g_{2}$ denote the renormalized top Yukawa, U(1) gauge, and SU(2) gauge couplings, respectively.

Taking a cut like in eq. (2.2) leads to what we term master spectral functions:

$$
\rho_{\mathcal{I}_{\mathrm{X}}} \equiv \operatorname{Im}\left[\mathcal{I}_{\mathbf{x}}\right]_{k_{n} \rightarrow-i\left[k_{0}+i 0^{+}\right]} .
$$

Numerical results for all of these are listed in appendix B, apart from $\rho_{\mathcal{I}_{\mathrm{j}}}$; the case $\rho_{\mathcal{I}_{\mathrm{j}}}$, together with the general methodology used, were discussed in ref. [15]. In the next section we collect the results obtained after inserting the master spectral functions into the imaginary part of eq. (2.6).

\section{Main results}

\subsection{Strict NLO expression}

Each of the master spectral functions can be split into two parts:

$$
\rho_{\mathcal{I}_{\mathrm{X}}}=\rho_{\mathcal{I}_{\mathrm{X}}}^{\mathrm{vac}}+\rho_{\mathcal{I}_{\mathrm{X}}}^{T}
$$

The first term must include all divergences, and may be chosen to include finite parts as well. We note that although denoted by $\rho_{\mathcal{I}_{\mathrm{x}}}^{\mathrm{vac}}$, this structure does have an overall temperature dependence, of the same functional form as the leading-order (LO) result which it renormalizes. The purely thermal part $\rho_{\mathcal{I}_{\mathrm{X}}}^{T}$ is, in contrast, finite and of a more complicated functional form. The divergences of the vacuum parts cancel against those in $\mathcal{Z}_{\nu}$, eq. (2.7). Subsequently, with the choices of $\rho_{\mathcal{I}_{\mathrm{X}}}^{\mathrm{vac}}$ explained in appendix B, we obtain a finite 


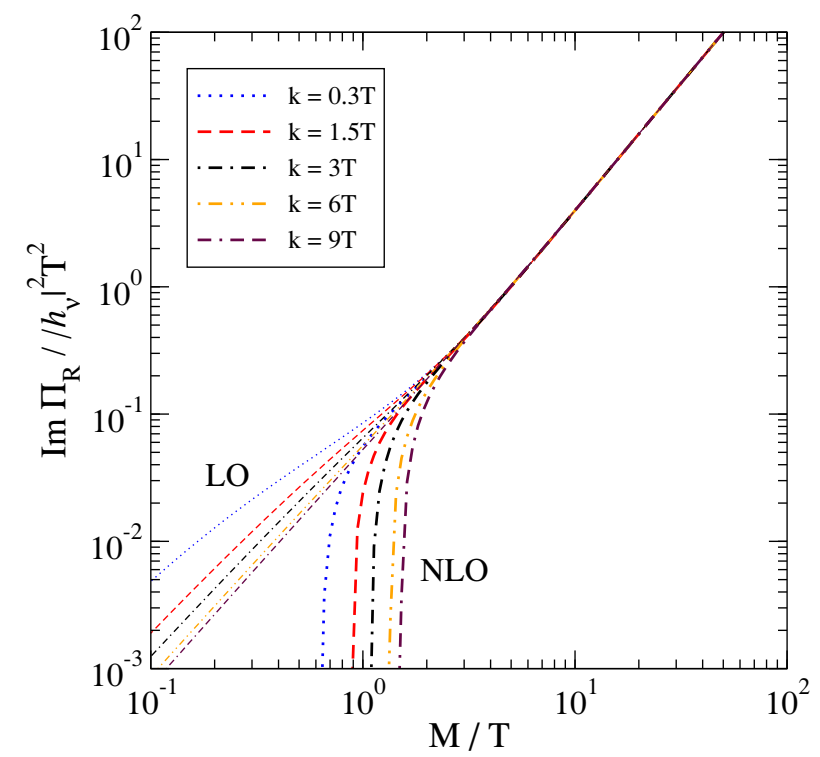

Figure 1. The expression from eq. (3.2), in units of $T^{2}$, for $k_{0}^{2} \equiv k^{2}+M^{2}$. The couplings and the renormalization scale are fixed as specified in appendix C. The loop expansion breaks down at $M \sim T$.

renormalized expression for the imaginary part of the retarded correlator:

$$
\begin{aligned}
\frac{\operatorname{Im} \Pi_{\mathrm{R}}}{\left|h_{\nu}(\bar{\mu})\right|^{2}}= & \frac{M^{2} T}{8 \pi k} \ln \left[\frac{\sinh \left(k_{+} / T\right)}{\sinh \left(k_{-} / T\right)}\right] \\
& +12 \lambda\left\{-\rho_{\mathcal{I}_{\mathrm{b}}}^{T}+\rho_{\mathcal{I}_{\mathrm{d}}}^{T}\right\} \\
& +2 h_{t}^{2} N_{\mathrm{c}}\left\{2\left[\rho_{\mathcal{I}_{\mathrm{b}}^{T}}^{T}-\rho_{\widetilde{\mathcal{I}}_{\mathrm{d}}^{T}}^{T}\right]-\rho_{\widetilde{\mathcal{I}}_{\mathrm{f}}}^{T}+\rho_{\widetilde{\mathcal{I}}_{\mathrm{h}}}^{T}\right. \\
& \left.-\frac{\pi M^{2}}{(4 \pi)^{4} k} \int_{k_{-}}^{k_{+}} \mathrm{d} p \frac{n_{\mathrm{F}}\left(k_{0}-p\right) n_{\mathrm{B}}(p)}{n_{\mathrm{F}}\left(k_{0}\right)}\left[\ln \frac{\left(k_{+}-p\right)\left(p-k_{-}\right) \bar{\mu}^{2}}{k^{2} M^{2}}+\frac{11}{2}\right]\right\} \\
& +\frac{g_{1}^{2}+3 g_{2}^{2}}{2}\left\{-\rho_{\mathcal{I}_{\mathrm{b}}}^{T}+2\left[-\rho_{\mathcal{I}_{\mathrm{b}}}^{T}+\rho_{\mathcal{I}_{\mathrm{d}}}^{T}-\rho_{\mathcal{I}_{\mathrm{d}}}^{T}\right]+3 \rho_{\mathcal{I}_{\mathrm{d}}}^{T}\right. \\
& +2\left[\rho_{\mathcal{I}_{\mathrm{g}}}^{T}+\rho_{\mathcal{\mathcal { I }}_{\mathrm{h}}}^{T}+\rho_{\mathcal{I}_{\mathrm{j}}}^{T}\right]-4\left[\rho_{\mathcal{I}_{\mathrm{h}}}^{T}+\rho_{\mathcal{\mathcal { I }}_{\mathrm{h}}}^{T}\right] \\
& \left.+\frac{3 \pi M^{2}}{(4 \pi)^{4} k} \int_{k_{-}}^{k_{+}} \mathrm{d} p \frac{n_{\mathrm{F}}\left(k_{0}-p\right) n_{\mathrm{B}}(p)}{n_{\mathrm{F}}\left(k_{0}\right)}\left[\ln \frac{\left(k_{+}-p\right)\left(p-k_{-}\right) \bar{\mu}^{2}}{k^{2} M^{2}}+\frac{41}{6}\right]\right\} .
\end{aligned}
$$

Here we have defined

$$
k_{ \pm} \equiv \frac{k_{0} \pm k}{2}, \quad M^{2} \equiv \mathcal{K}^{2}=4 k_{+} k_{-}>0,
$$

and $n_{\mathrm{B}}, n_{\mathrm{F}}$ are the Bose and Fermi distributions.

A numerical evaluation of this expression is shown in figure 1 (parameters and the renormalization scale are chosen as explained in appendix C). Results are displayed at several momenta on both sides of $k=3 T$. It is clear that the naive loop expansion breaks down for $M \sim T$. Based on this plot one might conclude that loop corrections decrease the production rate but, as will become apparent in section 3.3, such a conclusion is premature. 


\subsection{Non-relativistic limit}

In the non-relativistic limit $M^{2} \gg(\pi T)^{2}$ eq. (3.2) can be represented in more explicit form: up to and including $\mathcal{O}\left(T^{4} / M^{2}\right)$, this "OPE" expression reads [13]

$$
\begin{aligned}
\frac{\operatorname{Im} \Pi_{\mathrm{R}}}{\left|h_{\nu}(\bar{\mu})\right|^{2}}= & \frac{M^{2}}{8 \pi}\left\{1-\frac{12 \lambda}{M^{2}} \int_{\mathbf{p}} \frac{n_{\mathrm{B}}}{p}\right. \\
& -h_{t}^{2} N_{\mathrm{c}}\left[\frac{1}{(4 \pi)^{2}}\left(\ln \frac{\bar{\mu}^{2}}{M^{2}}+\frac{7}{2}\right)+\frac{k_{0}^{2}+k^{2} / 3}{M^{6}} \int_{\mathbf{p}} \frac{4 p n_{\mathrm{F}}}{3}\right] \\
& \left.+\left(g_{1}^{2}+3 g_{2}^{2}\right)\left[\frac{3}{4(4 \pi)^{2}}\left(\ln \frac{\bar{\mu}^{2}}{M^{2}}+\frac{29}{6}\right)+\frac{k_{0}^{2}+k^{2} / 3}{M^{6}} \int_{\mathbf{p}} \frac{p\left(17 n_{\mathrm{F}}-16 n_{\mathrm{B}}\right)}{3}\right]\right\}
\end{aligned}
$$

where the integrals over the phase space distributions have elementary forms:

$$
\int_{\mathbf{p}} \frac{n_{\mathrm{B}}}{p}=\frac{T^{2}}{12}, \quad \int_{\mathbf{p}} \frac{n_{\mathrm{F}}}{p}=\frac{T^{2}}{24}, \quad \int_{\mathbf{p}} p n_{\mathrm{B}}=\frac{\pi^{2} T^{4}}{30}, \quad \int_{\mathbf{p}} p n_{\mathrm{F}}=\frac{7 \pi^{2} T^{4}}{240} .
$$

An interesting question is how low the temperature should be in order for eq. (3.5) to yield an accurate representation of the full result. It has been pointed out in ref. [15] that for a particular 2-loop master spectral function, the non-relativistic approximation is only accurate for $M \gtrsim 25 T$. However, in eq. (3.2) the 2-loop contributions are suppressed by couplings and loop factors, whereas for the 1-loop term the thermal corrections are exponentially small for $k_{+}, k_{-} \gg \pi T$. Therefore, a somewhat better convergence may be expected.

The full and non-relativistic results are compared in figure 2 for $k=3 T$. We observe that the OPE-asymptotics appears to join the full expression at $M \gtrsim 4 T$. This is somewhat of an optical illusion, however; as shown by the right panel, even the sign of the thermal correction is correctly reproduced only for $M \gtrsim 10 T$, and the relative error decreases only for $M \gtrsim 15 T$. In any case the convergence is better in the full result than in the particular individual 2-loop master spectral function studied in ref. [15].

\subsection{Towards ultra-relativistic limit}

Let us return to the breakdown of the loop expansion, as illustrated in figure 1 . What happens is that for $k \sim \pi T$ but $M \ll \pi T$, i.e. $2 k_{-}=\sqrt{k^{2}+M^{2}}-k \approx M^{2} /(2 k) \ll$ $\pi T$, the LO term is of magnitude $\operatorname{Im} \Pi_{\mathrm{R}}^{\mathrm{LO}} /\left|h_{\nu}\right|^{2} \sim M^{2} \ln (T / M)$ whereas the NLO term is of magnitude $\operatorname{Im} \Pi_{\mathrm{R}}^{\mathrm{NLO}} /\left|h_{\nu}\right|^{2} \sim g^{2} M^{2} T^{2} / k_{-}^{2} \sim g^{2} T^{4} / M^{2}$, where $g^{2}$ denotes a generic coupling. The relative magnitude of the correction is $\sim g^{2} T^{4} / M^{4}$, and consequently the loop expansion requires resummation for $M \lesssim g^{\frac{1}{2}} T$. The most divergent NLO correction comes with a negative sign; it is related to Higgs mass thermal resummation, as will be discussed presently.

For an even smaller $M \lesssim g T$, further resummations are needed. A Hard Thermal Loop (HTL) resummation was presented in ref. [19], however HTL resummation alone does not lead to a consistent weak-coupling expansion for the present observable in the ultrarelativistic regime [6]. Indeed a systematic computation requires a Landau-Pomeranchuk-Migdal (LPM) resummation $[7,8]$. This amounts to a solution of a Schrödinger-type equation 

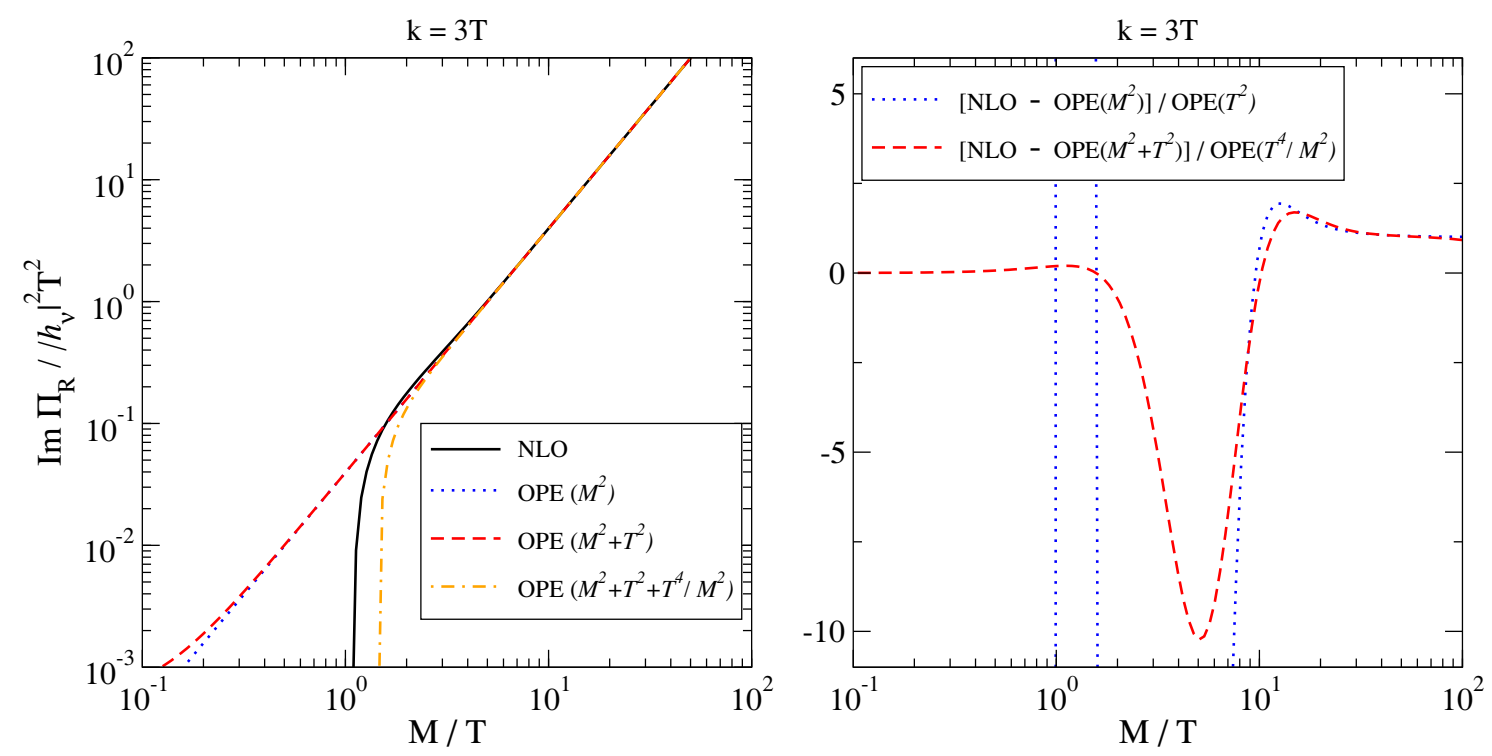

Figure 2. Left: the "OPE" expression from eq. (3.4), up to three consecutive orders as indicated in the parentheses, compared with the "NLO" result from eq. (3.2), for $k_{0}^{2} \equiv k^{2}+M^{2}$. Right: the relative difference between eqs. (3.2), (3.4). The couplings and the renormalization scale are fixed as specified in appendix C. On the resolution of the logarithmic plot it seems that the OPE expression is accurate for all $M \gtrsim 4 T$, however as shown by the right panel discrepancies with respect to the unexpanded expression are correctly represented only for $M \gtrsim 15 T$.

with a light-cone potential, which implements a resummation of ladder diagrams, representing multiple soft scatterings taking place within the average "formation time" of the ultrarelativistic right-handed neutrino being produced.

In the present study, we will not implement LPM resummation (comments on this are however made in section 6). Rather, we follow the convention of capturing a sub-series of higher order corrections through the assignment of thermal masses to otherwise massless particles. The concept of a thermal mass is ambiguous for particles of non-zero spin, depending e.g. on whether soft $(k \ll \pi T)$ or hard $(k \gtrsim \pi T)$ excitations are considered. For the present problem the latter kinematics is the relevant one, and then the thermal masses are those sometimes called the "asymptotic" ones (for a concise summary see ref. [20]). It turns out that the Higgs mass thermal resummation (where no ambiguities appear) indeed consistently removes the dominant divergences from $\operatorname{Im} \Pi_{\mathrm{R}}$ in the regime $M \sim g^{\frac{1}{2}} T$, as we now show.

Let us start by simply inserting the masses $m_{\phi}, m_{\ell}$ for the Higgs and for the left-handed leptons, respectively, and subsequently define a Euclidean correlator through

$$
\Pi_{\mathrm{E}}^{\text {tree }}(K) \equiv 4\left|h_{\nu}\right|^{2} \oiint_{P} \frac{K \cdot(P-K)}{\left[P^{2}+m_{\phi}^{2}\right]\left[(P-K)^{2}+m_{\ell}^{2}\right]} .
$$

This 1-loop sum-integral is labelled a "tree-level" contribution because of frequent conventions in literature: its cut corresponds to $1 \leftrightarrow 2$ scatterings. Carrying out the Matsubara sum and taking the cut, one finds three channels, for $M>m_{\phi}+m_{\ell}, m_{\phi}>M+m_{\ell}$, and 
$m_{\ell}>M+m_{\phi}$, respectively. For the actual values relevant for the Standard Model,

$$
\begin{aligned}
& m_{\phi}^{2}=\frac{T^{2}}{16}\left(g_{1}^{2}+3 g_{2}^{2}+\frac{4}{3} h_{t}^{2} N_{\mathrm{c}}+8 \lambda\right), \\
& m_{\ell}^{2}=\frac{T^{2}}{16}\left(g_{1}^{2}+3 g_{2}^{2}\right),
\end{aligned}
$$

only the first two channels can get realized. In each channel, the angular integral between the directions of $\mathbf{p}$ and $\mathbf{k}$ can be carried out by taking $E_{p k} \equiv \sqrt{(\mathbf{p}-\mathbf{k})^{2}+m_{\ell}^{2}}$ as an integration variable, and subsequently the integral over the radial direction can also be performed, by taking $E_{p} \equiv \sqrt{p^{2}+m_{\phi}^{2}}$ as a variable. For $k_{0}=\sqrt{k^{2}+M^{2}}$ the result reads ${ }^{1}$

$$
\begin{aligned}
\frac{\operatorname{Im} \Pi_{\mathrm{R}}^{\text {tree }}=}{\left|h_{\nu}\right|^{2}}= & \frac{\left(M^{2}-m_{\phi}^{2}+m_{\ell}^{2}\right) T}{8 \pi k} \ln \left\{\frac{\sinh \left(\frac{E_{\max }}{2 T}\right) \cosh \left(\frac{k_{0}-E_{\min }}{2 T}\right)}{\sinh \left(\frac{E_{\min }}{2 T}\right) \cosh \left(\frac{k_{0}-E_{\max }}{2 T}\right)}\right\} \\
& \times\left[\theta\left(M-m_{\phi}-m_{\ell}\right)-\theta\left(m_{\phi}-m_{\ell}-M\right)-\theta\left(m_{\ell}-m_{\phi}-M\right)\right],
\end{aligned}
$$

where

$$
\begin{aligned}
E_{\max (\min )} & \equiv \frac{k_{0}\left(M^{2}+m_{\phi}^{2}-m_{\ell}^{2}\right) \pm k \Delta\left(M, m_{\phi}, m_{\ell}\right)}{2 M^{2}} \\
\Delta\left(M, m_{\phi}, m_{\ell}\right) & \equiv \sqrt{M^{4}-2 M^{2}\left(m_{\phi}^{2}+m_{\ell}^{2}\right)+\left(m_{\phi}^{2}-m_{\ell}^{2}\right)^{2}} .
\end{aligned}
$$

Suppose now that we are in the regime $m_{\phi}^{2}, m_{\ell}^{2} \ll M^{2}$, and expand to first order in the small masses. Then

$$
E_{\min } \approx k_{-}+\frac{m_{\phi}^{2}}{4 k_{-}}-\frac{m_{\ell}^{2}}{4 k_{+}}, \quad E_{\max } \approx k_{+}+\frac{m_{\phi}^{2}}{4 k_{+}}-\frac{m_{\ell}^{2}}{4 k_{-}},
$$

and eq. (3.9) becomes

$$
\begin{aligned}
\frac{\operatorname{Im} \Pi_{\mathrm{R}}^{\text {tree }}}{\left|h_{\nu}\right|^{2}} \approx & \frac{\left(M^{2}-m_{\phi}^{2}+m_{\ell}^{2}\right) T}{8 \pi k} \ln \left\{\frac{\sinh \left(k_{+} / T\right)}{\sinh \left(k_{-} / T\right)}\right\} \\
& +\frac{M^{2}}{8 \pi k}\left\{m_{\phi}^{2}\left[\frac{1+n_{\mathrm{B}}\left(k_{+}\right)-n_{\mathrm{F}}\left(k_{-}\right)}{4 k_{+}}-\frac{1+n_{\mathrm{B}}\left(k_{-}\right)-n_{\mathrm{F}}\left(k_{+}\right)}{4 k_{-}}\right]\right. \\
& \left.+m_{\ell}^{2}\left[\frac{1+n_{\mathrm{B}}\left(k_{-}\right)-n_{\mathrm{F}}\left(k_{+}\right)}{4 k_{+}}-\frac{1+n_{\mathrm{B}}\left(k_{+}\right)-n_{\mathrm{F}}\left(k_{-}\right)}{4 k_{-}}\right]\right\}+\mathcal{O}\left(m_{\phi, \ell}^{4}\right) .
\end{aligned}
$$

Inserting the expressions from eqs. (3.7), (3.8) this is seen to agree exactly with the sum of all $\rho_{\mathcal{I}_{\mathrm{b}}}$ 's and $\rho_{\mathcal{I}_{\mathrm{d}}}$ 's in eq. (3.2), cf. eqs. (B.15), (B.22). These master spectral functions include the only quadratically divergent structures in the limit $k_{-} \ll \pi T$ as can be deduced from the right panels of figures $6-11$. The most divergent terms are $\sim M^{2} n_{\mathrm{B}}\left(k_{-}\right) / k_{-}$ and are related to the Higgs mass resummation as is clearly visible from the second row of eq. (3.13).

\footnotetext{
${ }^{1}$ Equivalent expressions can be found in literature.
} 


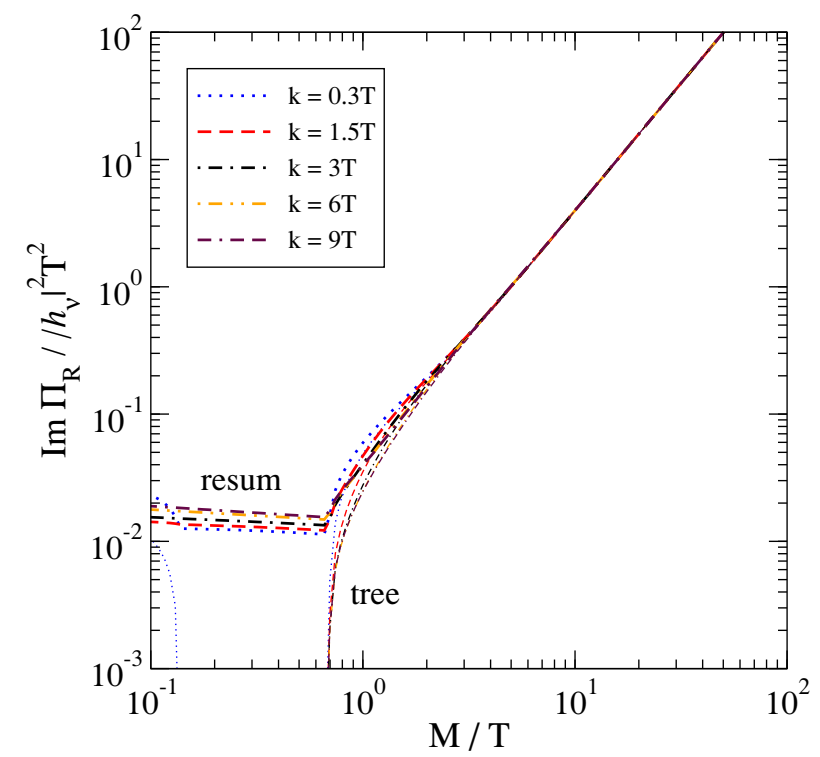

Figure 3. The mass-resummed expression from eq. (3.14) (thick lines), in units of $T^{2}$, for $k_{0}^{2} \equiv k^{2}+M^{2}$, versus the tree-level result from eq. (3.9) (thin lines). The couplings and the renormalization scale are fixed as specified in appendix C. The cusp is expected to be removed through higher-order corrections, but the overall magnitude of the mass-resummed result is already in qualitative agreement with the ultrarelativistic results of refs. [7, 8] (cf. figure 5).

We now define a "resummed" result by accounting for all $\rho_{\mathcal{I}_{\mathrm{b}}}$ 's and $\rho_{\mathcal{I}_{\mathrm{d}}}$ 's of eq. (3.2) through the thermal masses:

$$
\begin{aligned}
\frac{\operatorname{Im} \Pi_{\mathrm{R}}^{\text {resum }} \equiv}{\left|h_{\nu}(\bar{\mu})\right|^{2}} \equiv & \frac{\operatorname{Im} \Pi_{\mathrm{R}}^{\text {tree }}}{\left|h_{\nu}(\bar{\mu})\right|^{2}} \\
& +2 h_{t}^{2} N_{\mathrm{c}}\left\{-\rho_{\widetilde{\mathcal{I}}_{\mathrm{f}}^{T}}^{T}+\rho_{\widetilde{\mathcal{I}}_{\mathrm{h}}}^{T}\right. \\
& \left.-\frac{\pi M^{2}}{(4 \pi)^{4} k} \int_{k_{-}}^{k_{+}} \mathrm{d} p \frac{n_{\mathrm{F}}\left(k_{0}-p\right) n_{\mathrm{B}}(p)}{n_{\mathrm{F}}\left(k_{0}\right)}\left[\ln \frac{\left(k_{+}-p\right)\left(p-k_{-}\right) \bar{\mu}^{2}}{k^{2} M^{2}}+\frac{11}{2}\right]\right\} \\
& +\frac{g_{1}^{2}+3 g_{2}^{2}}{2}\left\{2\left[\rho_{\mathcal{I}_{\mathrm{g}}}^{T}+\rho_{\widehat{\mathcal{I}}_{\mathrm{h}}}^{T}+\rho_{\mathcal{I}_{\mathrm{j}}}^{T}\right]-4\left[\rho_{\mathcal{I}_{\mathrm{h}}}^{T}+\rho_{\widehat{\mathcal{I}}_{\mathrm{h}}^{T}}^{T}\right]\right. \\
& \left.+\frac{3 \pi M^{2}}{(4 \pi)^{4} k} \int_{k_{-}}^{k_{+}} \mathrm{d} p \frac{n_{\mathrm{F}}\left(k_{0}-p\right) n_{\mathrm{B}}(p)}{n_{\mathrm{F}}\left(k_{0}\right)}\left[\ln \frac{\left(k_{+}-p\right)\left(p-k_{-}\right) \bar{\mu}^{2}}{k^{2} M^{2}}+\frac{41}{6}\right]\right\},
\end{aligned}
$$

where $\operatorname{Im} \Pi_{\mathrm{R}}^{\text {tree }}$ is the tree-level result from eq. (3.9). Note that the remaining master spectral functions continue to be evaluated without masses (in these spectral functions masses amount to higher-order corrections).

The tree and mass-resummed spectral functions are shown in figure 3. It is apparent that the downwards divergence seen in figure 1 is a reflection of thermal mass generation; after this effect has been taken into account, the other NLO terms show an enhancement. However the mass resummation implemented does not capture all the terms that need to be resummed for $M \lesssim g T$; indeed, as has been demonstrated with the case of hot QCD [21] and more recently with the problem at hand $[7,8]$, cusps such as those seen in figure 3 

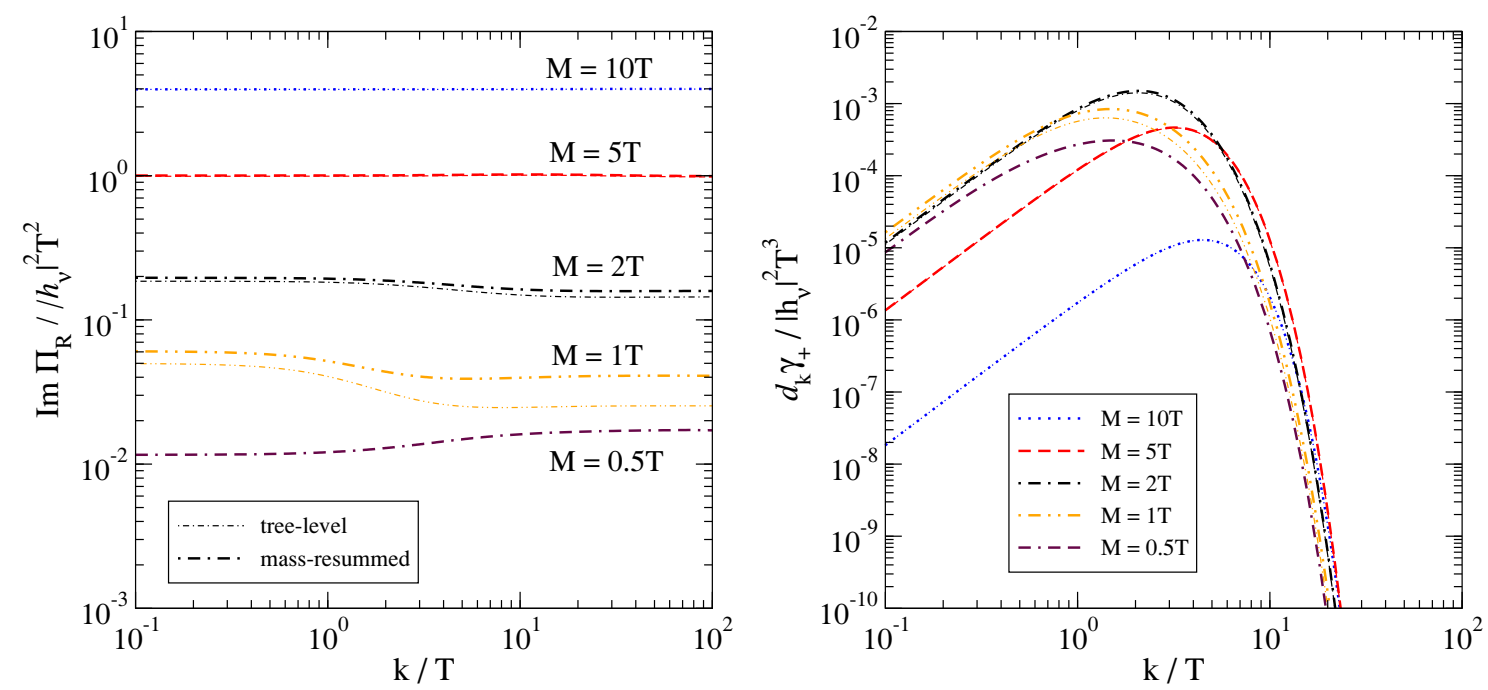

Figure 4. Left: the $k$-dependence of eq. (3.14) (thick lines), for selected $M$ and $k_{0}^{2} \equiv k^{2}+M^{2}$. Thin lines indicate the "tree-level" result from eq. (3.9). In the regime of validity of the computation, i.e. $M \gtrsim \pi T, k$-dependence is quite modest. Right: the corresponding production spectra, $\partial_{k} \gamma_{+}$ from eq. (4.1). The couplings and the renormalization scale are fixed as specified in appendix C.

are also removed through a systematic resummation of all corrections pertinent to the ultrarelativistic regime. ${ }^{2}$ It is nevertheless interesting that the order of magnitude of the mass-resummed result is not unlike that found in refs. [7, 8] (cf. section 5).

\section{Spectra and spectral functions}

We have already observed that, for a fixed $\mathcal{K}^{2}=M^{2}$, the non-covariant dependence of $\operatorname{Im} \Pi_{\mathrm{R}}$ on the spatial momentum $k$ is small, cf. figure 3. This is illustrated again in figure 4(left), for a number of different $M / T$. Subsequently we plot the whole spectra according to eq. (2.4), i.e.

$$
\partial_{k} \gamma_{+} \equiv \frac{k^{2} n_{\mathrm{F}}\left(\sqrt{k^{2}+M^{2}}\right)}{\pi^{2} \sqrt{k^{2}+M^{2}}} \operatorname{Im} \Pi_{\mathrm{R}}
$$

for a few selected $M / T$, in figure 4(right). Obviously the latter results display a much stronger $k$-dependence than $\operatorname{Im} \Pi_{R}$, however this emerges through the trivial "kinematic" structures shown in eq. (4.1), rather than complicated plasma physics determining $\operatorname{Im} \Pi_{R}$.

\section{Total production rate}

According to eq. (2.4), the total right-handed neutrino production rate reads

$$
\gamma_{+}=\int_{0}^{\infty} \mathrm{d} k \partial_{k} \gamma_{+}
$$

\footnotetext{
${ }^{2}$ The mass-resummed result of the current study still diverges logarithmically for $M / T \rightarrow 0$; this originates from the master spectral function $\rho_{\widehat{\mathcal{I}}_{\mathrm{h}}}^{T}$, cf. figure 11(right). The divergence is also removed by resummations.
} 


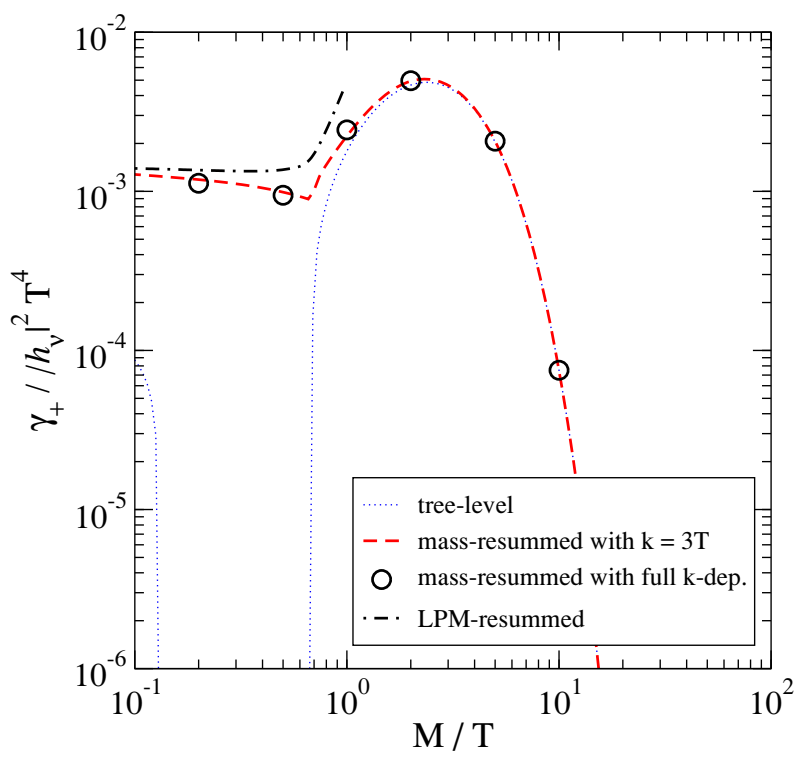

Figure 5. The total production rate based on evaluating $\operatorname{Im} \Pi_{\mathrm{R}}$ at $k=3 T$ and taking it as a constant otherwise (red dashed line), compared with results obtained with the full $k$-dependence included (black circles). The couplings and the renormalization scale are fixed as specified in appendix C. For $M \lesssim T$ we also compare with the complete LPM-resummed result from ref. [8] (dash-dotted line).

where $\partial_{k} \gamma_{+}$is the differential production rate from eq. (4.1). Given the small $k$-dependence as seen in figure 4(left), a good approximation for the total production rate can be obtained by evaluating the "expensive" Im $\Pi_{\mathrm{R}}$ only at some typical momentum, for instance $k \sim$ 3T. The accuracy of this approximation is illustrated in figure 5, and found to be in general excellent. It should be noted that in the non-relativistic regime the actual average momentum is $k \sim \sqrt{2 M T}$ rather than $k \sim 3 T$, but the approximation does not lose its accuracy, because the $k$-dependence of $\operatorname{Im} \Pi_{\mathrm{R}}$ becomes even less significant for $M \gg \pi T$ (cf. figure $4($ left)).

In figure 5 the total production rate is also compared with the LPM-resummed result from ref. [8]. Although our expression is not reliable for $M \ll \pi T$ and the result of ref. [8] is not reliable for $M \gtrsim \pi T$, it is remarkable how well the two appear to extrapolate towards each other. (Our results are closer to the systematic analysis of ref. [8] than the phenomenological approach of ref. [22].) In principle it should also be possible to combine the two results into an expression applicable for a general $M / \pi T$ (some comments are made in section 6), however implementing this in practice necessitates a dedicated separate study.

\section{Conclusions and outlook}

The purpose of this paper has been to extend previous NLO results for the right-handed neutrino production rate up to higher temperatures, into the so-called relativistic regime in which the temperature is of a similar magnitude as the mass of the right-handed neutrinos. In the so-called strong washout scenario of leptogenesis, the right-handed neutrinos 
equilibrate initially, whereby no lepton asymmetry exists in a certain temperature range; it is generated at low temperatures when the right-handed neutrinos chemically decouple and can subsequently decay. It is conceivable that most of the decays take place in a nonrelativistic regime, however it is not clear a priori how accurate computations based on the non-relativistic approximation are, because thermal effects are only power-suppressed [14]. The results obtained here suggest that corrections are substantial for $T \gtrsim M / 4$. If a significant contribution arises from this range, then the results of the current study may be used for a more precise analysis.

One finding of the current investigation is that in the relativistic and non-relativistic regimes, the non-covariant dependence of the retarded correlator denoted by $\operatorname{Im} \Pi_{R}$ on the spatial momentum with respect to the heat bath is quite modest (cf. figure 4(left)). Therefore the "expensive" part of the computation needs to be carried out only for a specific chosen $k$, for instance $k=3 T$, in order to determine the overall magnitude of $\operatorname{Im} \Pi_{\mathrm{R}}$. When inserted into the proper overall relations, this information is sufficient for determining the total production rate with good accuracy (cf. figure 5).

Apart from the strong washout scenario of leptogenesis, another possibility is that the right-handed neutrinos never equilibrate chemically; one then speaks of a weak washout scenario (cf. e.g. ref. [23]). In this case even ultrarelativistic temperatures play a role, and resummations are needed for consistent results [7, 8]. Although the results of the current paper lose their validity when approaching the ultrarelativistic regime, they do allow us to anticipate some features of the corresponding expressions, such as that there is no gap between the two possible $1 \leftrightarrow 2$ channels permitted by tree-level kinematics with thermal masses (cf. figure 3). In addition the total rates of the two approaches extrapolate towards each other surprisingly well, even though both computations eventually leave their ranges of validity (cf. figure 5). The physics conclusion from the enhanced rate at high temperatures is that chemical equilibration is more likely than naively expected, which may reduce the parameter space for weak-washout leptogenesis.

In principle, it should be possible to combine the current NLO results, valid in the nonrelativistic and relativistic regimes, with the resummed LO result of the ultrarelativistic regime $[7,8]$. Some care is however needed for avoiding double-counting in doing so. Matching computations of a necessary type have been carried out with HTL resummation previously, but the case of LPM resummation seems to represent a bigger challenge. ${ }^{3}$ Nevertheless the problem may be worth giving a go; for practical applications a result valid for all temperatures would clearly be quite convenient. (Ultimately NLO corrections should also be worked out for the LPM regime; they are likely to be suppressed only by $\sqrt{g^{2}}$ there [25].)

\footnotetext{
${ }^{3}$ Schematically, omitting Lorentz-violating structures, the NLO computation we have carried out is of the form $\operatorname{Im} \Pi_{\mathrm{R}, \mathrm{UV}} \sim M^{2}\left[\phi_{1}\left(T^{2} / M^{2}\right)+g^{2} \phi_{2}\left(T^{2} / M^{2}\right)+\ldots\right]$ whereas any resummed result contains all orders in $g^{2}: \operatorname{Im} \Pi_{\mathrm{R}, \mathrm{IR}} \sim M^{2}\left[\chi_{1}\left(T^{2} / M^{2}, g^{2}\right)+\ldots\right]$. For combining the two, the resummed result has to be re-expanded in $g^{2}$, in order to cancel the terms from $\operatorname{Im} \Pi_{\mathrm{R}, \mathrm{UV}}$ that it resums: $\operatorname{Im} \Pi_{\mathrm{R}, \text { full }} \sim$ $M^{2}\left\{\chi_{1}\left(T^{2} / M^{2}, g^{2}\right)+\phi_{1}\left(T^{2} / M^{2}\right)-\chi_{1}\left(T^{2} / M^{2}, 0\right)+g^{2}\left[\phi_{2}\left(T^{2} / M^{2}\right)-\partial_{g^{2}} \chi_{1}\left(T^{2} / M^{2}, 0\right)\right]+\ldots\right\}$. The determination of the latter subtraction term is not quite trivial, because $\chi_{1}\left(T^{2} / M^{2}, g^{2}\right)$ contains parts only available through a numerical solution of an inhomogeneous Schrödinger-type equation and is otherwise complicated as well.
} 
Another possible direction for future research is the application of the methods and master spectral functions discussed here to other problems of cosmological relevance. For instance, the determination of the gravitino production rate from a hot Standard Model plasma is a problem perhaps meriting a further look.

\section{Acknowledgments}

I am grateful to Dietrich Bödeker for helpful discussions. This work was partly supported by the Swiss National Science Foundation (SNF) under grant 200021-140234.

\section{A Definitions of master sum-integrals}

Denoting by $\mathscr{\&}_{P}$ and $\mathscr{E}_{\{P\}}$ sum-integrals over bosonic and fermionic Matsubara fourmomenta, the master sum-integrals entering the computation are defined as follows [13]:

$$
\begin{aligned}
& \mathcal{J}_{\mathrm{a}} \equiv \sum_{P} \frac{1}{P^{2}}, \\
& \widetilde{\mathcal{J}}_{\mathrm{a}} \equiv \sum_{\{P\}} \frac{1}{P^{2}}, \\
& \mathcal{J}_{\mathrm{b}} \equiv \oint_{P} \frac{K^{2}}{P^{2}(P-K)^{2}}, \\
& \mathcal{I}_{\mathrm{b}} \equiv \varlimsup_{P Q} \frac{1}{Q^{2} P^{2}(P-K)^{2}} \text {, } \\
& \widetilde{\mathcal{I}}_{\mathrm{b}} \equiv \oint_{P\{Q\}} \frac{1}{Q^{2} P^{2}(P-K)^{2}} \text {, } \\
& \mathcal{I}_{\mathrm{c}} \equiv \sum_{P Q} \frac{1}{Q^{2} P^{4}}, \\
& \widetilde{\mathcal{I}}_{\mathrm{c}} \equiv \sum_{P\{Q\}} \frac{1}{Q^{2} P^{4}}, \\
& \widehat{\mathcal{I}}_{\mathrm{c}} \equiv \sum_{\{P\} Q} \frac{1}{Q^{2} P^{4}} \text {, } \\
& \overline{\mathcal{I}}_{\mathrm{c}} \equiv \sum_{\{P Q\}} \frac{1}{Q^{2} P^{4}}, \\
& \mathcal{I}_{\mathrm{d}} \equiv \sum_{P Q} \frac{K^{2}}{Q^{2} P^{4}(P-K)^{2}} \text {, } \\
& \widetilde{\mathcal{I}}_{\mathrm{d}} \equiv \varlimsup_{P\{Q\}} \frac{K^{2}}{Q^{2} P^{4}(P-K)^{2}} \text {, } \\
& \widehat{\mathcal{I}}_{\mathrm{d}} \equiv \sum_{\{P\} Q} \frac{K^{2}}{Q^{2} P^{4}(P-K)^{2}} \text {, }
\end{aligned}
$$




$$
\begin{aligned}
& \overline{\mathcal{I}}_{\mathrm{d}} \equiv \varlimsup_{\{P Q\}} \frac{K^{2}}{Q^{2} P^{4}(P-K)^{2}} \text {, } \\
& \mathcal{I}_{\mathrm{e}} \equiv \overbrace{P Q} \frac{1}{Q^{2} P^{2}(P-Q)^{2}} \text {, } \\
& \widetilde{\mathcal{I}}_{\mathrm{e}} \equiv \sum_{P\{Q\}} \frac{1}{Q^{2} P^{2}(P-Q)^{2}} \text {, } \\
& \mathcal{I}_{\mathrm{f}} \equiv \lim _{\lambda \rightarrow 0} \mathcal{F}_{P Q} \frac{1}{Q^{2}\left[(Q-P)^{2}+\lambda^{2}\right](P-K)^{2}}, \\
& \widetilde{\mathcal{I}}_{\mathrm{f}} \equiv \lim _{\lambda \rightarrow 0} \sum_{P\{Q\}} \frac{1}{Q^{2}\left[(Q-P)^{2}+\lambda^{2}\right](P-K)^{2}}, \\
& \mathcal{I}_{\mathrm{g}} \equiv \oint_{P Q} \frac{K^{2}}{P^{2}(P-K)^{2} Q^{2}(Q-K)^{2}} \text {, } \\
& \mathcal{I}_{\mathrm{h}} \equiv \lim _{\lambda \rightarrow 0} \oint_{P Q} \frac{K^{2}}{Q^{2} P^{2}\left[(Q-P)^{2}+\lambda^{2}\right](P-K)^{2}} \text {, } \\
& \widetilde{\mathcal{I}}_{\mathrm{h}} \equiv \lim _{\lambda \rightarrow 0} \oint_{P\{Q\}} \frac{K^{2}}{Q^{2} P^{2}\left[(Q-P)^{2}+\lambda^{2}\right](P-K)^{2}}, \\
& \widehat{\mathcal{I}}_{\mathrm{h}} \equiv \lim _{\lambda \rightarrow 0} \oint_{\{P\} Q} \frac{K^{2}}{Q^{2} P^{2}\left[(Q-P)^{2}+\lambda^{2}\right](P-K)^{2}}, \\
& \widehat{\mathcal{I}}_{\mathrm{h}}, \equiv \lim _{\lambda \rightarrow 0} \oint_{\{P\} Q} \frac{2 K \cdot Q}{Q^{2} P^{2}\left[(Q-P)^{2}+\lambda^{2}\right](P-K)^{2}}, \\
& \mathcal{I}_{\mathrm{j}} \equiv \lim _{\lambda \rightarrow 0} \oint_{P Q} \frac{K^{4}}{Q^{2} P^{2}\left[(Q-P)^{2}+\lambda^{2}\right](P-K)^{2}(Q-K)^{2}} \text {. }
\end{aligned}
$$

In order to handle the different statistics simultaneously, we introduce a generic labelling of lines (with individual propagators omitted or doubled in some cases):

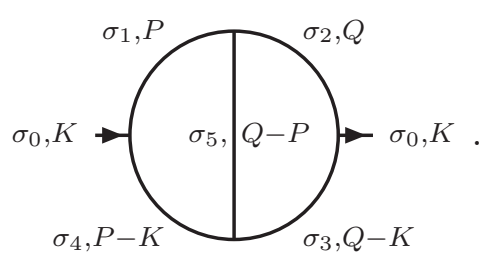

The labels $\sigma_{0}, \ldots, \sigma_{5}$ equal +1 for bosons and -1 for fermions. Given fermion number conservation, only two of the indices are independent, and the triple $\left(\sigma_{1} \sigma_{4} \sigma_{5}\right)$ has been chosen for this task; subsequently

$$
\sigma_{0}=\sigma_{1} \sigma_{4}, \quad \sigma_{2}=\sigma_{1} \sigma_{5}, \quad \sigma_{3}=\sigma_{4} \sigma_{5}
$$

The spectral functions are obtained from eq. (2.8) which is commensurate with the spectral representation

$$
\int_{-\infty}^{\infty} \frac{\mathrm{d} k_{0}}{\pi} \frac{\rho_{\mathcal{I}_{\mathrm{x}}}}{k_{0}-i k_{n}}=\mathcal{I}_{\mathrm{x}} .
$$


In practice, the spectral function can be read from an imaginary-time correlator by partial fractioning its dependence on $k_{n}$ and by then replacing

$$
\frac{1}{-i k_{n}+C} \rightarrow \pi \delta\left(-k_{0}+C\right) \text {. }
$$

Note that structures containing no or polynomial $k_{n}^{2}$-dependence yield a vanishing spectral function according to eq. (2.8); in these cases eq. (A.26) needs to be modified by "contact terms" (a discussion can be found e.g. in ref. [14]).

\section{B Results for master spectral functions in time-like domain}

In the following spectral functions are listed for the range $k_{0}>k>0$. Results for $k_{0} \rightarrow-k_{0}$ follow from antisymmetry, whereas the space-like domain $0<k_{0}<k$ has not been worked out here, although thermal spectral functions can be non-zero there as well.

\section{B.1 $\rho_{\mathcal{J}_{\mathrm{a}}}$}

Since $\mathcal{J}_{\mathrm{a}}$ and $\widetilde{\mathcal{J}}_{\mathrm{a}}$ are independent of the external momentum, there is no cut:

$$
\rho_{\mathcal{J}_{\mathrm{a}}}=\rho_{\widetilde{\mathcal{J}}_{\mathrm{a}}}=0
$$

\section{B.2 $\rho_{\mathcal{J}_{\mathrm{b}}}$}

For the case in eq. (A.3) we get, after carrying out the Matsubara sum,

$$
\begin{aligned}
\mathcal{J}_{\mathrm{b}}= & \int_{\mathbf{p}} \frac{K^{2}}{4 \epsilon_{p} \epsilon_{p k}}\left\{\left[\frac{1}{i k_{n}+\epsilon_{p}+\epsilon_{p k}}+\frac{1}{-i k_{n}+\epsilon_{p}+\epsilon_{p k}}\right]\left[1+n_{\sigma_{1}}\left(\epsilon_{p}\right)+n_{\sigma_{4}}\left(\epsilon_{p k}\right)\right]\right. \\
& \left.+\left[\frac{1}{i k_{n}-\epsilon_{p}+\epsilon_{p k}}+\frac{1}{-i k_{n}-\epsilon_{p}+\epsilon_{p k}}\right]\left[n_{\sigma_{1}}\left(\epsilon_{p}\right)-n_{\sigma_{4}}\left(\epsilon_{p k}\right)\right]\right\}
\end{aligned}
$$

where we used the labelling of eq. (A.24), denoted

$$
\epsilon_{p} \equiv p \equiv|\mathbf{p}|, \quad \epsilon_{p k} \equiv|\mathbf{p}-\mathbf{k}|,
$$

and defined

$$
n_{\sigma}(\epsilon) \equiv \frac{\sigma}{e^{\epsilon / T}-\sigma}, \quad n_{\sigma}^{-1}(\epsilon)=\sigma e^{\epsilon / T}-1, \quad \int^{\epsilon} \mathrm{d} \epsilon^{\prime} n_{\sigma}\left(\epsilon^{\prime}\right)=T \ln \left(1-\sigma e^{-\epsilon / T}\right),
$$

which satisfies $n_{+}=n_{\mathrm{B}}$ and $n_{-}=-n_{\mathrm{F}}$.

Taking the cut, only one of the four channels contributes for $k_{0}>k$, and the spectral function reads

$$
\rho_{\mathcal{J}_{\mathrm{b}}}=-\int_{\mathbf{p}} \frac{\pi \mathcal{K}^{2}}{4 \epsilon_{p} \epsilon_{p k}} \delta\left(k_{0}-\epsilon_{p}-\epsilon_{p k}\right)\left[1+n_{\sigma_{1}}\left(\epsilon_{p}\right)+n_{\sigma_{4}}\left(\epsilon_{p k}\right)\right] .
$$

It is often convenient to employ the alternative representation

$$
1+n_{\sigma_{1}}\left(\epsilon_{p}\right)+n_{\sigma_{4}}\left(k_{0}-\epsilon_{p}\right)=n_{\sigma_{0}}^{-1}\left(k_{0}\right) n_{\sigma_{4}}\left(k_{0}-p\right) n_{\sigma_{1}}(p),
$$

where we made use of $\sigma_{1} \sigma_{4}=\sigma_{0}$. 
Because the leading-order contribution gets multiplied by a counterterm, we need to determine it up to $\mathcal{O}(\epsilon)$. The integration measure reads, in $d=3-2 \epsilon$ spatial dimensions,

$$
\int_{\mathbf{p}}=\frac{(4 \pi)^{\epsilon}}{4 \pi^{2} \Gamma(1-\epsilon)} \int_{0}^{\infty} \mathrm{d} p p^{2-2 \epsilon} \int_{-1}^{+1} \mathrm{~d} z\left(1-z^{2}\right)^{-\epsilon},
$$

where $z \equiv \mathbf{p} \cdot \mathbf{k} / p k$. The integral over $z$ can be converted into one over $\epsilon_{p k}$ through

$$
\mathrm{d} z=-\frac{\epsilon_{p k} \mathrm{~d} \epsilon_{p k}}{p k},
$$

and the Dirac- $\delta$ gets realized for $k_{-}<p<k_{+}$, with $k_{ \pm}$defined according to eq. (3.3). Recalling the constraint $\delta\left(k_{0}-p-\epsilon_{p k}\right)$, the function appearing in the angular integration is conveniently expressed as

$$
1-z^{2}=\frac{\mathcal{K}^{2}\left(k_{+}-p\right)\left(p-k_{-}\right)}{k^{2} p^{2}} .
$$

Introducing the $\overline{\mathrm{MS}}$ scheme scale parameter, $\bar{\mu}$, by inserting

$$
1=\mu^{-2 \epsilon} \bar{\mu}^{2 \epsilon} \frac{e^{\epsilon \gamma_{E}}}{(4 \pi)^{\epsilon}},
$$

and suppressing the inconsequential $\mu^{-2 \epsilon}$, we thereby obtain

$$
\begin{aligned}
\rho_{\mathcal{J}_{\mathrm{b}}}= & -\frac{\pi \mathcal{K}^{2}}{(4 \pi)^{2} k} \frac{e^{\epsilon \gamma_{\mathrm{E}}}}{\Gamma(1-\epsilon)} \int_{k_{-}}^{k_{+}} \mathrm{d} p \frac{n_{\sigma_{4}}\left(k_{0}-p\right) n_{\sigma_{1}}(p)}{n_{\sigma_{0}}\left(k_{0}\right)}\left[\frac{\bar{\mu}^{2} k^{2}}{\mathcal{K}^{2}\left(k_{+}-p\right)\left(p-k_{-}\right)}\right]^{\epsilon} \\
= & -\frac{\pi \mathcal{K}^{2}}{(4 \pi)^{2} k} \int_{k_{-}}^{k_{+}} \mathrm{d} p \frac{n_{\sigma_{4}}\left(k_{0}-p\right) n_{\sigma_{1}}(p)}{n_{\sigma_{0}}\left(k_{0}\right)} \\
& \times\left[1+\epsilon \ln \frac{\bar{\mu}^{2}}{\mathcal{K}^{2}}+\epsilon \ln \frac{k^{2}}{\left(k_{+}-p\right)\left(p-k_{-}\right)}+\mathcal{O}\left(\epsilon^{2}\right)\right] .
\end{aligned}
$$

The remaining integral is easily carried out in the term of $\mathcal{O}\left(\epsilon^{0}\right)$ :

$$
\begin{aligned}
\rho_{\mathcal{J}_{\mathrm{b}}}= & -\frac{\pi \mathcal{K}^{2}}{(4 \pi)^{2} k}\left\{T \ln \left(\frac{e^{k_{+} / T}+\sigma_{0} e^{-k_{+} / T}-\sigma_{1}-\sigma_{4}}{e^{k_{-} / T}+\sigma_{0} e^{-k_{-} / T}-\sigma_{1}-\sigma_{4}}\right)\left[1+\epsilon\left(\ln \frac{\bar{\mu}^{2}}{\mathcal{K}^{2}}+2\right)\right]\right. \\
& \left.+\epsilon \int_{k_{-}}^{k_{+}} \mathrm{d} p \frac{n_{\sigma_{4}}\left(k_{0}-p\right) n_{\sigma_{1}}(p)}{n_{\sigma_{0}}\left(k_{0}\right)}\left[\ln \frac{k^{2}}{\left(k_{+}-p\right)\left(p-k_{-}\right)}-2\right]\right\}+\mathcal{O}\left(\epsilon^{2}\right) .
\end{aligned}
$$

For $k_{+}, k_{-} \gg \pi T$, the second row vanishes up to exponentially small corrections so that, in accordance with ref. [13],

$$
\rho_{\mathcal{J}_{\mathrm{b}}} \stackrel{k_{+}, k_{-} \gg \pi T}{\approx}-\frac{\mathcal{K}^{2}}{16 \pi}\left[1+\epsilon\left(\ln \frac{\bar{\mu}^{2}}{\mathcal{K}^{2}}+2\right)+\mathcal{O}\left(\epsilon^{2}\right)\right]
$$

The specific statistics relevant for the current paper are

$$
\mathcal{J}_{\mathrm{b}} \Leftrightarrow\left(\sigma_{1} \sigma_{4} \sigma_{5} \mid \sigma_{0}\right)=(+-+\mid-) .
$$

Here and in the following, the values of selected non-independent indices as obtained from eq. (A.25) have also been indicated to the right of the vertical line. 

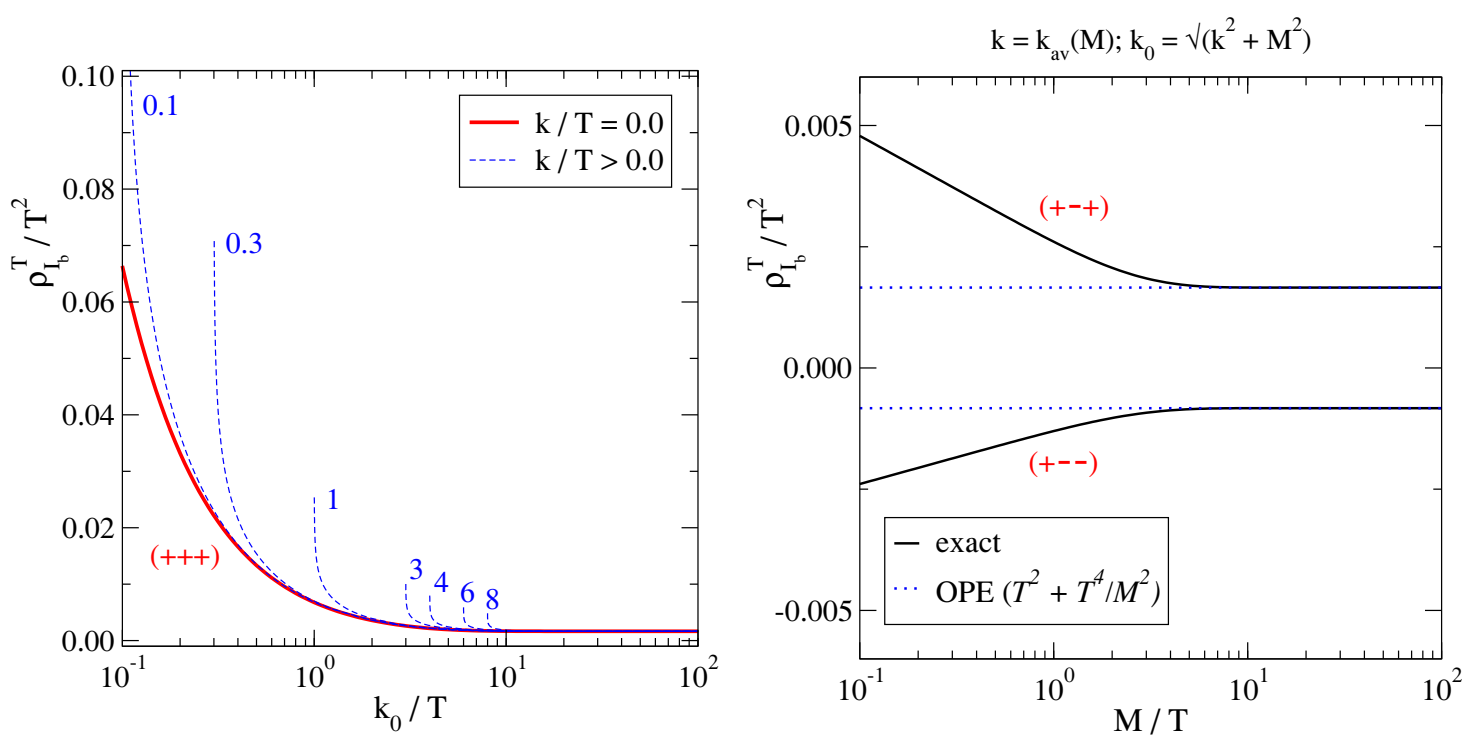

Figure 6. Left: the spectral function $\rho_{\mathcal{I}_{\mathrm{b}}}^{T} \equiv \rho_{\mathcal{I}_{\mathrm{b}}}$ with the purely bosonic statistics $\left(\sigma_{1} \sigma_{4} \sigma_{5}\right)=$ $(+++)$, for $k_{0} \geq k+0.001 T$, compared with the zero-momentum limit determined in ref. [24]. Right: the spectral function $\rho_{\mathcal{I}_{\mathrm{b}}}$ with the momentum of eq. (B.19) and statistics of eqs. (B.17), (B.18) as a function of $M / T$, compared with the OPE-asymptotics from eq. (B.16).

B.3 $\rho_{\mathcal{I}_{\mathrm{b}}}$

The spectral function corresponding to eq. (A.4) can be written generically as

$$
\rho_{\mathcal{I}_{\mathrm{b}}}=-\frac{\rho_{\mathcal{J}_{\mathbf{b}}}}{\mathcal{K}^{2}} \int_{\mathbf{q}} \frac{n_{\sigma_{2}}(q)}{q}=\frac{T}{16 \pi k} \ln \left(\frac{e^{k_{+} / T}+\sigma_{0} e^{-k_{+} / T}-\sigma_{1}-\sigma_{4}}{e^{k_{-} / T}+\sigma_{0} e^{-k_{-} / T}-\sigma_{1}-\sigma_{4}}\right) \int_{\mathbf{q}} \frac{n_{\sigma_{2}}(q)}{q} .
$$

For $k_{+}, k_{-} \gg \pi T$ this goes over into [13]

$$
\rho_{\mathcal{I}_{\mathrm{b}}} \stackrel{k_{+}, k_{-} \gg \pi T}{\approx} \frac{1}{16 \pi} \int_{\mathbf{q}} \frac{n_{\sigma_{2}}(q)}{q},
$$

up to exponentially small corrections. The value of the remaining integral is given in eq. (3.5). The specific statistics for the current problem are

$$
\begin{aligned}
& \mathcal{I}_{\mathrm{b}} \Leftrightarrow\left(\sigma_{1} \sigma_{4} \sigma_{5} \mid \sigma_{2} \sigma_{0}\right)=(+-+\mid+-), \\
& \widetilde{\mathcal{I}}_{\mathrm{b}} \Leftrightarrow\left(\sigma_{1} \sigma_{4} \sigma_{5} \mid \sigma_{2} \sigma_{0}\right)=(+--\mid--) .
\end{aligned}
$$

Defining a typical thermal momentum through

$$
k_{\mathrm{av}}^{2}(M) \equiv \frac{\int_{0}^{\infty} \mathrm{d} k k^{4} \exp \left(-\frac{\sqrt{k^{2}+M^{2}}}{T}\right)}{\int_{0}^{\infty} \mathrm{d} k k^{2} \exp \left(-\frac{\sqrt{k^{2}+M^{2}}}{T}\right)}=\frac{3 M T K_{3}\left(\frac{M}{T}\right)}{K_{2}\left(\frac{M}{T}\right)},
$$

numerical results for $\rho_{\mathcal{I}_{\mathrm{b}}}$ are shown in figure 6 .

B.4 $\rho_{\mathcal{I}_{\mathrm{c}}}$

Since all versions of $\mathcal{I}_{\mathrm{c}}$ are independent of the external momentum, there is no cut:

$$
\rho_{\mathcal{I}_{\mathrm{C}}}=\rho_{\widetilde{\mathcal{I}}_{\mathrm{C}}}=\rho_{\widehat{\mathcal{I}}_{\mathrm{C}}}=\rho_{\overline{\mathcal{I}}_{\mathrm{C}}}=0 .
$$




\section{B.5 $\rho_{\mathcal{I}_{\mathrm{d}}}$}

The derivation of the spectral function corresponding to eq. (A.10) follows from that for $\rho_{\mathcal{J}_{\mathrm{d}}}$ in section B.2; we simply give the line with momentum $P$ a mass, $\lambda$, and take a derivative with respect to the mass. If we change variables from $p$ to

$$
E_{p} \equiv \sqrt{p^{2}+\lambda^{2}},
$$

then $\lambda$ only appears in the boundaries of the $E_{p}$-integration. No terms of $\mathcal{O}(\epsilon)$ are needed, so the general result can be expressed as

$$
\begin{aligned}
\rho_{\mathcal{I}_{\mathrm{d}}} & =\frac{\pi \mathcal{K}^{2}}{(4 \pi)^{2} k} \int_{\mathbf{q}} \frac{n_{\sigma_{2}}(q)}{q} \frac{\mathrm{d}}{\mathrm{d} \lambda^{2}}\left\{\int_{k_{-}+\frac{\lambda^{2}}{4 k_{-}}}^{k_{+}+\frac{\lambda^{2}}{4 k_{+}}} \mathrm{d} E_{p}\left[1+n_{\sigma_{1}}\left(E_{p}\right)+n_{\sigma_{4}}\left(k_{0}-E_{p}\right)\right]\right\}_{\lambda=0} \\
& =\frac{\pi \mathcal{K}^{2} n_{\sigma_{0}}^{-1}\left(k_{0}\right)}{(4 \pi)^{2} k}\left[\frac{n_{\sigma_{1}}\left(k_{+}\right) n_{\sigma_{4}}\left(k_{-}\right)}{4 k_{+}}-\frac{n_{\sigma_{1}}\left(k_{-}\right) n_{\sigma_{4}}\left(k_{+}\right)}{4 k_{-}}\right] \int_{\mathbf{q}} \frac{n_{\sigma_{2}}(q)}{q}
\end{aligned}
$$

The value of the remaining integral is given in eq. (3.5). For $k_{+}, k_{-} \gg \pi T$ the asymptotics reads [13]

$$
\rho_{\mathcal{I}_{\mathrm{d}}} \stackrel{k_{+}, k_{-} \gg \pi T}{\approx}-\frac{1}{16 \pi} \int_{\mathbf{q}} \frac{n_{\sigma_{2}}(q)}{q}
$$

with exponentially small corrections. The specific statistics for the current problem are

$$
\begin{aligned}
& \mathcal{I}_{\mathrm{d}} \Leftrightarrow\left(\sigma_{1} \sigma_{4} \sigma_{5} \mid \sigma_{2} \sigma_{0}\right)=(+-+\mid+-), \\
& \widetilde{\mathcal{I}}_{\mathrm{d}} \Leftrightarrow\left(\sigma_{1} \sigma_{4} \sigma_{5} \mid \sigma_{2} \sigma_{0}\right)=(+--\mid--), \\
& \widehat{\mathcal{I}}_{\mathrm{d}} \Leftrightarrow\left(\sigma_{1} \sigma_{4} \sigma_{5} \mid \sigma_{2} \sigma_{0}\right)=(-+-\mid+-), \\
& \overline{\mathcal{I}}_{\mathrm{d}} \Leftrightarrow\left(\sigma_{1} \sigma_{4} \sigma_{5} \mid \sigma_{2} \sigma_{0}\right)=(-++\mid--) .
\end{aligned}
$$

A numerical evaluation in shown in figure 7.

\section{B.6 $\rho_{\mathcal{I}_{\mathrm{e}}}$}

Since both versions of $\mathcal{I}_{\mathrm{e}}$ are independent of the external momentum, there is no cut:

$$
\rho_{\mathcal{I}_{\mathrm{e}}}=\rho_{\widetilde{\mathcal{I}}_{\mathrm{e}}}=0 .
$$



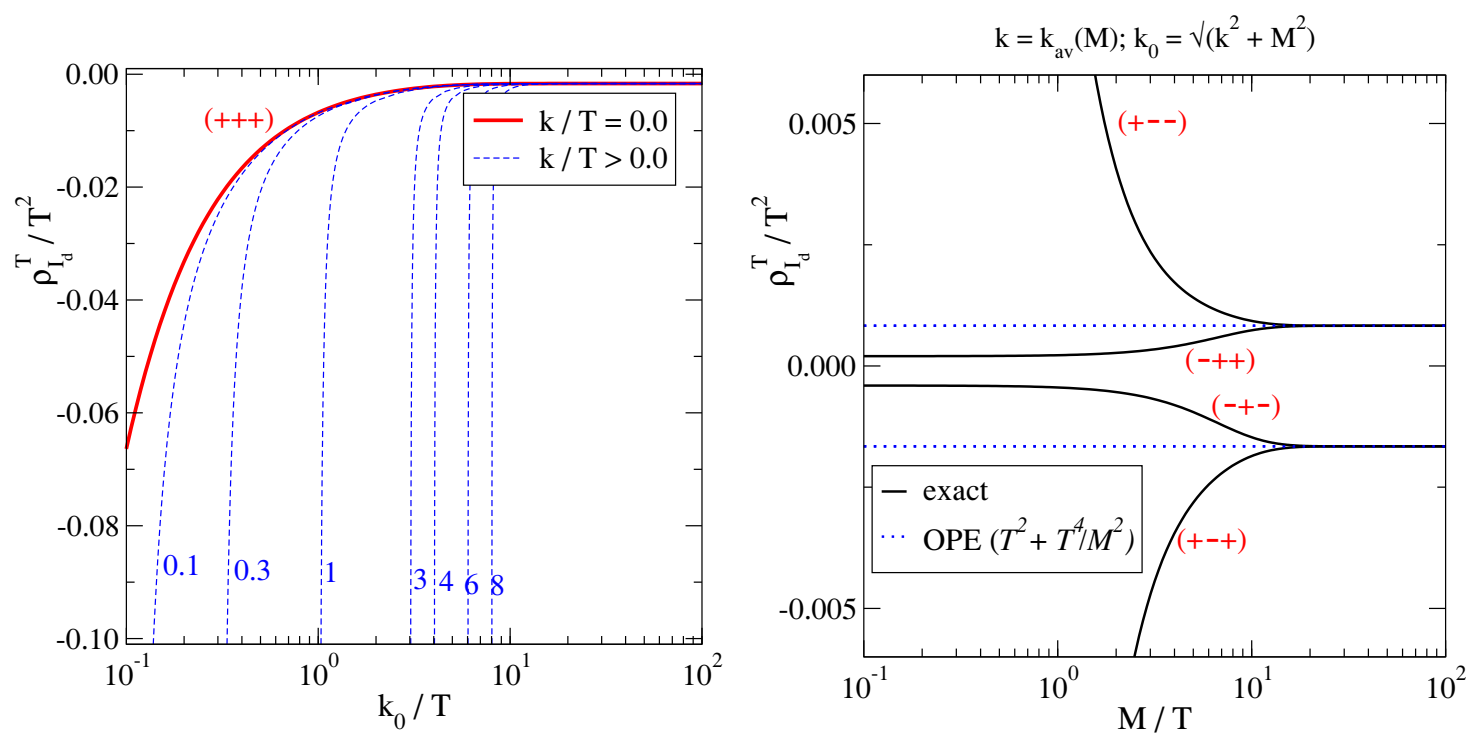

Figure 7. Left: the spectral function $\rho_{\mathcal{I}_{\mathrm{d}}} \equiv \rho_{\mathcal{I}_{\mathrm{d}}}^{T}$ with the purely bosonic statistics $\left(\sigma_{1} \sigma_{4} \sigma_{5}\right)=$ $(+++)$, for $k_{0} \geq k+0.001 T$, compared with the zero-momentum limit determined in ref. [24]. Right: the spectral function $\rho_{\mathcal{I}_{\mathrm{d}}}$ with the momentum of eq. (B.19) and statistics of eqs. (B.24)-(B.27) as a function of $M / T$, compared with the OPE-asymptotics from eq. (B.23).

\section{B.7 $\rho_{\mathcal{I}_{\mathrm{f}}}$}

After carrying out the Matsubara sums, the expression for $\mathcal{I}_{\mathrm{f}}$ reads

$$
\begin{aligned}
\mathcal{I}_{\mathrm{f}}= & \lim _{\lambda \rightarrow 0} \int_{\mathbf{p}, \mathbf{q}} \frac{1}{8 \epsilon_{q} \epsilon_{p k} E_{q p}}\{ \\
& \frac{1}{-i k_{n}+\epsilon_{p k}+\epsilon_{q}+E_{q p}}\left(\left[1+n_{\sigma_{4}}\left(\epsilon_{p k}\right)+n_{\sigma_{2}}\left(\epsilon_{q}\right)\right]\left[1+n_{\sigma_{5}}\left(E_{q p}\right)\right]+n_{\sigma_{4}}\left(\epsilon_{p k}\right) n_{\sigma_{2}}\left(\epsilon_{q}\right)\right) \\
& +\frac{1}{-i k_{n}-\epsilon_{p k}+\epsilon_{q}+E_{q p}}\left(n_{\sigma_{4}}\left(\epsilon_{p k}\right)\left[1+n_{\sigma_{2}}\left(\epsilon_{q}\right)+n_{\sigma_{5}}\left(E_{q p}\right)\right]-n_{\sigma_{2}}\left(\epsilon_{q}\right) n_{\sigma_{5}}\left(E_{q p}\right)\right) \\
& +\frac{1}{-i k_{n}+\epsilon_{p k}-\epsilon_{q}+E_{q p}}\left(n_{\sigma_{2}}\left(\epsilon_{q}\right)\left[1+n_{\sigma_{4}}\left(\epsilon_{p k}\right)+n_{\sigma_{5}}\left(E_{q p}\right)\right]-n_{\sigma_{4}}\left(\epsilon_{p k}\right) n_{\sigma_{5}}\left(E_{q p}\right)\right) \\
& \left.+\frac{1}{-i k_{n}+\epsilon_{p k}+\epsilon_{q}-E_{q p}}\left(n_{\sigma_{5}}\left(E_{q p}\right)\left[1+n_{\sigma_{4}}\left(\epsilon_{p k}\right)+n_{\sigma_{2}}\left(\epsilon_{q}\right)\right]-n_{\sigma_{4}}\left(\epsilon_{p k}\right) n_{\sigma_{2}}\left(\epsilon_{q}\right)\right)\right\} \\
& +\left(i k_{n} \rightarrow-i k_{n}\right) .
\end{aligned}
$$

The corresponding spectral function is obtained from eq. (A.27). In addition to the energies of eq. (B.3), a further variable appears here which contains the infrared regulator $\lambda$ :

$$
E_{q p} \equiv \sqrt{(\mathbf{q}-\mathbf{p})^{2}+\lambda^{2}} .
$$

The four channels of eq. (B.29) represent real corrections and were labelled (r1)-(r4) in ref. [15]; the $2 \leftrightarrow 2$ and $1 \leftrightarrow 3$ scatterings that they represent are of the type illustrated in figure 3 of ref. [15] but with internal propagators carrying the indices $\sigma_{1}, \sigma_{3}$ shrunk to points. 
In the notation of ref. [15], eq. (4.28) now reads

$$
\begin{aligned}
\left\langle\Phi_{\mathrm{r} 1}\left(k_{0}-p_{0}|q| p_{0}-q \mid \cdot\right)\right\rangle & =-\left\langle\Phi_{\mathrm{r} 2}\left(p_{0}-k_{0}|q| p_{0}-q \mid \cdot\right)\right\rangle \\
& =-\left\langle\Phi_{\mathrm{r} 3}\left(k_{0}-p_{0}|-q| p_{0}-q \mid \cdot\right)\right\rangle \\
& =-\left\langle\Phi_{\mathrm{r} 4}\left(k_{0}-p_{0}|q| q-p_{0} \mid \cdot\right)\right\rangle \\
& =\frac{n_{\sigma_{4}}\left(k_{0}-p_{0}\right) n_{\sigma_{2}}(q) n_{\sigma_{5}}\left(p_{0}-q\right)}{2 n_{\sigma_{0}}\left(k_{0}\right)} .
\end{aligned}
$$

The integration over $p$, with ranges as specified in ref. [15], is trivial. Factoring out

$$
\frac{\pi n_{\sigma_{4}}\left(k_{0}-p\right) n_{\sigma_{2}}(q) n_{\sigma_{5}}(p-q)}{(4 \pi)^{4} k n_{\sigma_{0}}\left(k_{0}\right)}
$$

the $\lambda \rightarrow 0$ limits (in the different regimes as specified in ref. [15]) read:

$$
\begin{aligned}
& \text { (a) }=(\mathrm{l}): 4(p-q) \text {, } \\
& \text { (b) : } 4\left(k_{+}-q\right) \text {, } \\
& \text { (b) : } 4\left(p-k_{-}\right) \text {, } \\
& (\mathrm{c})=-(\mathrm{h})=-(\underline{\mathrm{h}})=-(\mathrm{j}): 4\left(k_{+}-k_{-}\right) \text {, }
\end{aligned}
$$

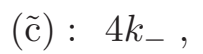

$$
\begin{aligned}
& \text { (d): } 4\left(k_{+}+q-p\right) \text {, } \\
& (\mathrm{e})=(\mathrm{f}): 4\left(k_{+}+k_{-}-p\right) \text {, } \\
& (\underline{\mathrm{e}})=(\underline{\mathrm{f}}): 4 q \text {, } \\
& (\mathrm{g})=(\underline{\mathrm{g}}): 4\left(k_{-}+q-p\right) \text {, } \\
& \text { (i) }=(\underline{\mathrm{k}}): 4\left(k_{-}-q\right) \text {, } \\
& (\mathrm{i})=(\mathrm{k}): 4\left(p-k_{+}\right) \text {. }
\end{aligned}
$$

For $k_{+}, k_{-} \gg \pi T$, the ultraviolet asymptotics of the spectral function reads [13]

$$
\rho_{\mathcal{I}_{\mathrm{f}}} \stackrel{k_{+}, k_{-} \gg \pi T}{\approx} \frac{\pi \mathcal{K}^{2}}{(4 \pi)^{4}} \times \frac{1}{2}+\int_{\mathbf{p}}\left\{\frac{n_{\sigma_{4}}+n_{\sigma_{5}}+n_{\sigma_{2}}}{16 \pi p}\right\}
$$

The specific statistics for the current problem are

$$
\begin{aligned}
& \mathcal{I}_{\mathrm{f}} \Leftrightarrow\left(\sigma_{1} \sigma_{4} \sigma_{5} \mid \sigma_{2} \sigma_{0}\right)=(+-+\mid+-), \\
& \widetilde{\mathcal{I}}_{\mathrm{f}} \Leftrightarrow\left(\sigma_{1} \sigma_{4} \sigma_{5} \mid \sigma_{2} \sigma_{0}\right)=(+--\mid--) .
\end{aligned}
$$

For numerical evaluation, we have reflected the final integral to the domain defined in figure 6 of ref. [15]. The corresponding integrand is not shown explicitly, since no substantial cancellations take place in the reflections. In addition, we always separate a "vacuum-like" part as in eq. (3.1), with a coefficient given by the leading term in eq. (B.44):

$$
\rho_{\mathcal{I}_{\mathrm{f}}}^{\mathrm{vac}} \equiv \frac{\pi \mathcal{K}^{2} T}{(4 \pi)^{4} k} \ln \left(\frac{e^{k_{+} / T}+\sigma_{0} e^{-k_{+} / T}-\sigma_{1}-\sigma_{4}}{e^{k_{-} / T}+\sigma_{0} e^{-k_{-} / T}-\sigma_{1}-\sigma_{4}}\right) \times \frac{1}{2} .
$$

It is the thermal part which is plotted numerically in figure 8 , and compared with the OPE asymptotics from eq. (B.44) as well as with the $k \rightarrow 0$ limit from ref. [24]. 

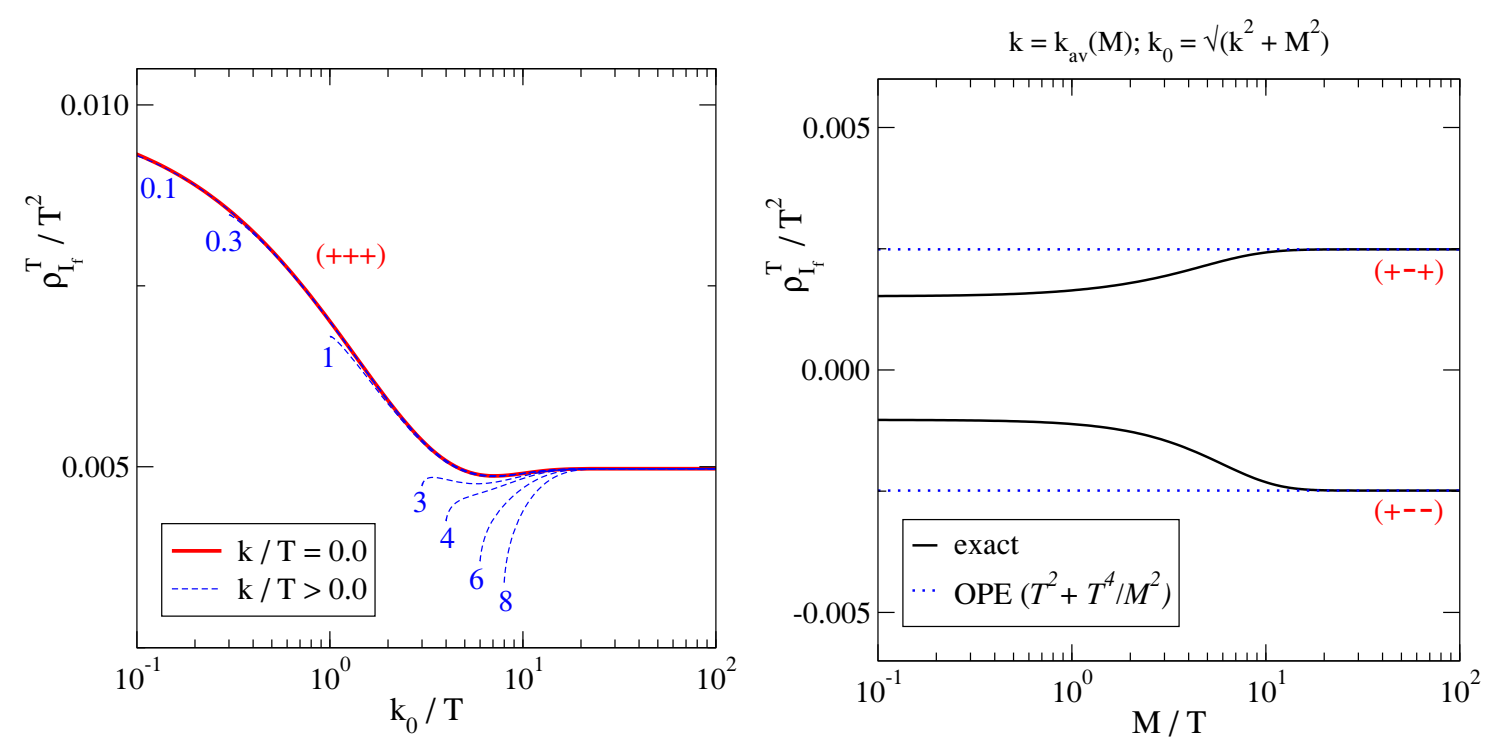

Figure 8. Left: the thermal part of $\rho_{\mathcal{I}_{\mathrm{f}}}$ with the purely bosonic statistics $\left(\sigma_{1} \sigma_{4} \sigma_{5}\right)=(+++)$, for $k_{0} \geq k+0.001 T$, compared with the zero-momentum limit determined in ref. [24]. Right: the thermal part of $\rho_{\mathcal{I}_{\mathrm{f}}}$ with the momentum of eq. (B.19) and statistics of eqs. (B.45), (B.46) as a function of $M / T$, compared with the OPE-asymptotics from eq. (B.44).

\section{B.8 $\rho_{\mathcal{I}_{\mathrm{g}}}$}

For $\mathcal{I}_{\mathrm{g}}$ of eq. (A.18), Matsubara sums lead to

$$
\begin{aligned}
& \mathcal{I}_{\mathrm{g}}=\int_{\mathbf{p}} \frac{K^{2}}{4 \epsilon_{p} \epsilon_{p k}}\{ {\left[\frac{1}{i k_{n}+\epsilon_{p}+\epsilon_{p k}}+\frac{1}{-i k_{n}+\epsilon_{p}+\epsilon_{p k}}\right]\left[1+n_{\sigma_{1}}\left(\epsilon_{p}\right)+n_{\sigma_{4}}\left(\epsilon_{p k}\right)\right] } \\
&+ {\left.\left[\frac{1}{i k_{n}-\epsilon_{p}+\epsilon_{p k}}+\frac{1}{-i k_{n}-\epsilon_{p}+\epsilon_{p k}}\right]\left[n_{\sigma_{1}}\left(\epsilon_{p}\right)-n_{\sigma_{4}}\left(\epsilon_{p k}\right)\right]\right\} } \\
& \times \int_{\mathbf{q}} \frac{1}{4 \epsilon_{q} \epsilon_{q k}}\{ {\left[\frac{1}{i k_{n}+\epsilon_{q}+\epsilon_{q k}}+\frac{1}{-i k_{n}+\epsilon_{q}+\epsilon_{q k}}\right]\left[1+n_{\sigma_{2}}\left(\epsilon_{q}\right)+n_{\sigma_{3}}\left(\epsilon_{q k}\right)\right] } \\
&\left.+\left[\frac{1}{i k_{n}-\epsilon_{q}+\epsilon_{q k}}+\frac{1}{-i k_{n}-\epsilon_{q}+\epsilon_{q k}}\right]\left[n_{\sigma_{2}}\left(\epsilon_{q}\right)-n_{\sigma_{3}}\left(\epsilon_{q k}\right)\right]\right\} .
\end{aligned}
$$

Taking the cut like in eq. (A.27), the result factorizes into a product of a structure like in eq. (B.5), and a principal value integral over the other part; the latter corresponds to a virtual loop correction, of the type illustrated in figure 3 of ref. [15] but with the line carrying the index $\sigma_{5}$ shrunk to a point. It has a divergent vacuum contribution,

$$
\int_{\mathbf{q}} \frac{1}{4 \epsilon_{q} \epsilon_{q k}} \mathbb{P}\left[\frac{1}{k_{0}+\epsilon_{q}+\epsilon_{q k}}+\frac{1}{-k_{0}+\epsilon_{q}+\epsilon_{q k}}\right]=\frac{1}{(4 \pi)^{2}}\left(\frac{1}{\epsilon}+\ln \frac{\bar{\mu}^{2}}{\mathcal{K}^{2}}+2\right),
$$

as well as a finite thermal part. In the latter the angular integral is doable if we substitute integration variables so as to always have $\epsilon_{p}$ or $\epsilon_{q}$ as the argument of the phase space 

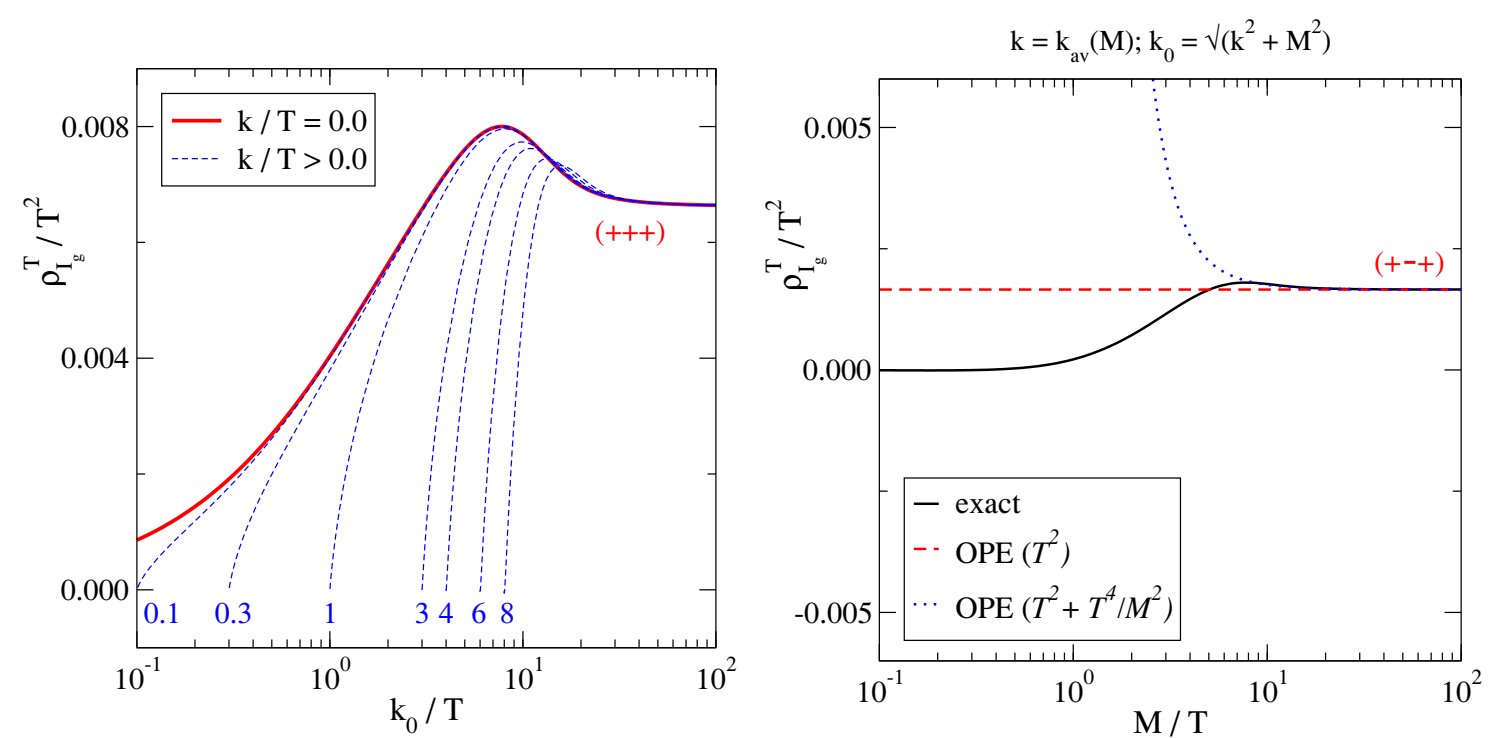

Figure 9. Left: the thermal part of $\rho_{\mathcal{I}_{\mathrm{g}}}$ (eq. (B.51)) with the purely bosonic statistics $\left(\sigma_{1} \sigma_{4} \sigma_{5}\right)=$ $(+++)$, for $k_{0} \geq k+0.001 T$, compared with the zero-momentum limit determined in ref. [24]. Right: the thermal part of $\rho_{\mathcal{I}_{\mathrm{g}}}$ with the momentum of eq. (B.19) and statistics of eq. (B.53) as a function of $M / T$, compared with the OPE-asymptotics from eq. (B.52).

distribution. Recalling the $\mathcal{O}(\epsilon)$-part from eq. (B.12), we get

$$
\begin{aligned}
\rho_{\mathcal{I}_{\mathrm{g}}}^{\mathrm{vac}} \equiv & -\frac{\pi \mathcal{K}^{2} T}{(4 \pi)^{4} k} \ln \left(\frac{e^{k_{+} / T}+\sigma_{0} e^{-k_{+} / T}-\sigma_{1}-\sigma_{4}}{e^{k_{-} / T}+\sigma_{0} e^{-k_{-} / T}-\sigma_{1}-\sigma_{4}}\right)\left(\frac{1}{\epsilon}+2 \ln \frac{\bar{\mu}^{2}}{\mathcal{K}^{2}}+4\right) \\
& +\left(\sigma_{1} \leftrightarrow \sigma_{2}, \sigma_{4} \leftrightarrow \sigma_{3}\right), \\
\rho_{\mathcal{I}_{\mathrm{g}}}^{T}= & \frac{\pi \mathcal{K}^{2}}{(4 \pi)^{4} k} \int_{k_{-}}^{k_{+}} \mathrm{d} p \frac{n_{\sigma_{4}}\left(k_{0}-p\right) n_{\sigma_{1}}(p)}{n_{\sigma_{0}}\left(k_{0}\right)}\left\{\ln \frac{\left(k_{+}-p\right)\left(p-k_{-}\right)}{k^{2}}+2\right. \\
& \left.+\int_{0}^{\infty} \frac{\mathrm{d} q}{k}\left(n_{\sigma_{2}}+n_{\sigma_{3}}\right)(q) \ln \left|\frac{\left(q-k_{+}\right)\left(q+k_{-}\right)}{\left(q+k_{+}\right)\left(q-k_{-}\right)}\right|\right\} \\
& +\left(\sigma_{1} \leftrightarrow \sigma_{2}, \sigma_{4} \leftrightarrow \sigma_{3}\right) .
\end{aligned}
$$

For $k_{+}, k_{-} \gg \pi T$, the ultraviolet asymptotics reads [13]

$$
\rho_{\mathcal{I}_{\mathrm{g}}} \stackrel{k_{+}, k_{-} \gg \pi T}{\approx}-\frac{\mathcal{K}^{2}}{2(4 \pi)^{3}}\left(\frac{1}{\epsilon}+2 \ln \frac{\bar{\mu}^{2}}{\mathcal{K}^{2}}+4\right)+\int_{\mathbf{p}} \sum_{i=1}^{4}\left\{\frac{n_{\sigma_{i}}}{16 \pi p}+\frac{p n_{\sigma_{i}}}{4 \pi} \frac{k_{0}^{2}+\frac{k^{2}}{3}}{\mathcal{K}^{4}}\right\} .
$$

The specific statistics for the current case are

$$
\mathcal{I}_{\mathrm{g}} \Leftrightarrow\left(\sigma_{1} \sigma_{4} \sigma_{5} \mid \sigma_{2} \sigma_{3} \sigma_{0}\right)=(+-+\mid+--) .
$$

The thermal part of $\rho_{\mathcal{I}_{\mathrm{g}}}$ is plotted numerically in figure 9 , and compared with the OPEasymptotics from eq. (B.52) as well as with the $k \rightarrow 0$ limit from ref. [24].

\section{B.9 $\rho_{\mathcal{I}_{\mathrm{h}}}$}

The spectral functions corresponding to $\mathcal{I}_{\mathrm{h}}, \widetilde{\mathcal{I}}_{\mathrm{h}}$, and $\widehat{\mathcal{I}}_{\mathrm{h}}$ can be handled simultaneously with the labelling of eq. (A.24), where now the line with the index $\sigma_{3}$ is absent. The expression 
after carrying out the Matsubara sums reads

$$
\begin{aligned}
\mathcal{I}_{\mathbf{h}}= & \lim _{\lambda \rightarrow 0} \int_{\mathbf{p}, \mathbf{q}} \frac{K^{2}}{8 \epsilon_{q} \epsilon_{p k} E_{q p}}\left\{\begin{array}{c}
{\left[1+n_{\sigma_{4}}\left(\epsilon_{p k}\right)+n_{\sigma_{2}}\left(\epsilon_{q}\right)\right]\left[1+n_{\sigma_{5}}\left(E_{q p}\right)\right]+n_{\sigma_{4}}\left(\epsilon_{p k}\right) n_{\sigma_{2}}\left(\epsilon_{q}\right)} \\
\epsilon_{p}^{2}-\left(\epsilon_{q}+E_{q p}\right)^{2}
\end{array}\right. \\
& \frac{1}{-i k_{n}+\epsilon_{p k}+\epsilon_{q}+E_{q p}} \frac{n_{\sigma_{4}}\left(\epsilon_{p k}\right)\left[1+n_{\sigma_{2}}\left(\epsilon_{q}\right)+n_{\sigma_{5}}\left(E_{q p}\right)\right]-n_{\sigma_{2}}\left(\epsilon_{q}\right) n_{\sigma_{5}}\left(E_{q p}\right)}{\epsilon_{p}^{2}-\left(\epsilon_{q}+E_{q p}\right)^{2}} \\
& +\frac{1}{-i k_{n}-\epsilon_{p k}+\epsilon_{q}+E_{q p}} \frac{n_{\sigma_{2}}\left(\epsilon_{q}\right)\left[1+n_{\sigma_{4}}\left(\epsilon_{p k}\right)+n_{\sigma_{5}}\left(E_{q p}\right)\right]-n_{\sigma_{4}}\left(\epsilon_{p k}\right) n_{\sigma_{5}}\left(E_{q p}\right)}{\epsilon_{p k}^{2}-\left(\epsilon_{q}-E_{q p}\right)^{2}} \\
& +\frac{1}{-i k_{n}+\epsilon_{p k}+\epsilon_{q}-E_{q p}} \frac{n_{\sigma_{5}}\left(E_{q p}\right)\left[1+n_{\sigma_{2}}\left(\epsilon_{q}\right)+n_{\sigma_{4}}\left(\epsilon_{p k}\right)\right]-n_{\sigma_{2}}\left(\epsilon_{q}\right) n_{\sigma_{4}}\left(\epsilon_{p k}\right)}{\epsilon_{p}^{2}-\left(\epsilon_{q}-E_{q p}\right)^{2}} \\
+ & \lim _{\lambda \rightarrow 0} \int_{\mathbf{p}} \frac{K^{2}}{4 \epsilon_{p} \epsilon_{p k}}\left[\frac{1+n_{\sigma_{1}}\left(\epsilon_{p}\right)+n_{\sigma_{4}}\left(\epsilon_{p k}\right)}{-i k_{n}+\epsilon_{p}+\epsilon_{p k}}+\frac{n_{\sigma_{1}}\left(\epsilon_{p}\right)-n_{\sigma_{4}}\left(\epsilon_{p k}\right)}{i k_{n}-\epsilon_{p}+\epsilon_{p k}}\right] \\
& \times \int_{\mathbf{q}}\left[\frac{1+n_{\sigma_{2}}\left(\epsilon_{q}\right)+n_{\sigma_{5}}\left(E_{q p}\right)}{4 \epsilon_{q} E_{q p}}\left(\frac{1}{\epsilon_{p}+\epsilon_{q}+E_{q p}}-\frac{1}{\epsilon_{p}-\epsilon_{q}-E_{q p}}\right)\right. \\
& \left.+\frac{n_{\sigma_{2}}\left(\epsilon_{q}\right)-n_{\sigma_{5}}\left(E_{q p}\right)}{4 \epsilon_{q} E_{q p}}\left(\frac{1}{\epsilon_{p}-\epsilon_{q}+E_{q p}}-\frac{1}{\epsilon_{p}+\epsilon_{q}-E_{q p}}\right)\right] \\
+ & \left(i k_{n} \rightarrow-i k_{n}\right) .
\end{aligned}
$$

Taking the cut like in eq. (A.27), the first four structures here are real corrections, the last two are virtual corrections. The corresponding scattering processes can be depicted like in figure 3 of ref. [15], with internal propagators carrying the index $\sigma_{3}$ shrunk to points.

For the real corrections, eq. (4.28) of ref. [15] now reads

$$
\begin{aligned}
\left\langle\Phi_{\mathrm{r} 1}\left(k_{0}-p_{0}|q| p_{0}-q \mid \cdot\right)\right\rangle & =-\left\langle\Phi_{\mathrm{r} 2}\left(p_{0}-k_{0}|q| p_{0}-q \mid \cdot\right)\right\rangle \\
& =-\left\langle\Phi_{\mathrm{r} 3}\left(k_{0}-p_{0}|-q| p_{0}-q \mid \cdot\right)\right\rangle \\
& =-\left\langle\Phi_{\mathrm{r} 4}\left(k_{0}-p_{0}|q| q-p_{0} \mid \cdot\right)\right\rangle \\
& =\frac{n_{\sigma_{4}}\left(k_{0}-p_{0}\right) n_{\sigma_{2}}(q) n_{\sigma_{5}}\left(p_{0}-q\right)}{n_{\sigma_{0}}\left(k_{0}\right)} \mathbb{P}\left\{\frac{\mathcal{K}^{2}}{2\left(p_{0}^{2}-p^{2}\right)}\right\} .
\end{aligned}
$$

The integration over $p$, with ranges as specified in ref. [15], is readily carried out, leading to simple logarithms. Renaming subsequently $p_{0} \rightarrow p$, and factoring out

$$
\frac{\pi \mathcal{K}^{2} n_{\sigma_{4}}\left(k_{0}-p\right) n_{\sigma_{2}}(q) n_{\sigma_{5}}(p-q)}{(4 \pi)^{4} k n_{\sigma_{0}}\left(k_{0}\right)},
$$


the $\lambda \rightarrow 0$ limits (in the sense specified in ref. [15]) read:

$$
\begin{aligned}
& \text { (a) }=(1): \frac{1}{p} \ln \left|\frac{4 p(p-q)^{2}}{\lambda^{2} q}\right| \text {, } \\
& \text { (b) : } \frac{1}{p} \ln \left|\frac{k_{+}(p-q)}{q\left(p-k_{+}\right)}\right| \text {, } \\
& \text { ( } \underline{\mathrm{b}}): \frac{1}{p} \ln \left|\frac{4 p(p-q)\left(p-k_{-}\right)}{\lambda^{2} k_{-}}\right| \text {, } \\
& (\mathrm{c})=-(\mathrm{h})=-(\underline{\mathrm{h}})=-(\mathrm{j}): \frac{1}{p} \ln \left|\frac{k_{+}\left(p-k_{-}\right)}{k_{-}\left(p-k_{+}\right)}\right| \text {, } \\
& (\tilde{\mathrm{c}}): \frac{1}{p} \ln \left|\frac{4 k_{-} p(p-q)}{\lambda^{2}\left(p-k_{-}\right)}\right|, \\
& \text {(d) : } \frac{1}{p} \ln \left|\frac{q k_{+}}{(p-q)\left(p-k_{+}\right)}\right| \text {, } \\
& (\mathrm{e})=(\mathrm{f}): \frac{1}{p} \ln \left|\frac{k_{+} k_{-}}{\left(p-k_{+}\right)\left(p-k_{-}\right)}\right| \text {, } \\
& (\underline{\mathrm{e}})=(\underline{\mathrm{f}}): \frac{1}{p} \ln \left|\frac{4 p q}{\lambda^{2}}\right|, \\
& (\mathrm{g})=(\underline{\mathrm{g}}): \frac{1}{p} \ln \left|\frac{q k_{-}}{(p-q)\left(p-k_{-}\right)}\right|, \\
& (\mathrm{i})=(\underline{\mathrm{k}}): \frac{1}{p} \ln \left|\frac{k_{-}(p-q)}{q\left(p-k_{-}\right)}\right| \text {, } \\
& (\underline{\mathrm{i}})=(\mathrm{k}): \frac{1}{p} \ln \left|\frac{4 p(p-q)\left(p-k_{+}\right)}{\lambda^{2} k_{+}}\right| \text {. }
\end{aligned}
$$

As far as the virtual corrections go, they include a divergent part:

$$
\begin{aligned}
\rho_{\mathcal{I}_{\mathrm{h}}} & \ni \int_{\mathbf{p}} \frac{\pi \mathcal{K}^{2} \delta\left(k_{0}-\epsilon_{p}-\epsilon_{p k}\right) n_{\sigma_{4}}\left(k_{0}-p\right) n_{\sigma_{1}}(p)}{4 \epsilon_{p} \epsilon_{p k} n_{\sigma_{0}}\left(k_{0}\right)} \int_{\mathbf{q}} \mathbb{P}\left\{\frac{1}{2 \epsilon_{q} E_{q p}} \frac{\epsilon_{q}+E_{q p}}{\epsilon_{p}^{2}-\left(\epsilon_{q}+E_{q p}\right)^{2}}\right\} \\
& =\rho_{\mathcal{J}_{\mathrm{b}}} \times\left.\operatorname{Re} \int_{Q} \frac{1}{Q^{2}\left[(Q-P)^{2}+\lambda^{2}\right]}\right|_{p_{n}=-i \epsilon_{p}} .
\end{aligned}
$$

Here we made use of the fact that the vacuum integral is independent of $p$ :

$$
\left.\operatorname{Re} \int_{Q} \frac{1}{Q^{2}\left[(Q-P)^{2}+\lambda^{2}\right]}\right|_{p_{n}=-i \epsilon_{p}}=\frac{1}{(4 \pi)^{2}}\left(\frac{1}{\epsilon}+\ln \frac{\bar{\mu}^{2}}{\lambda^{2}}+1\right) .
$$

It is helpful, however, not to separate the vacuum integral from the outset, but rather to treat the structures $\frac{1}{2}+n_{\sigma_{2}}\left(\epsilon_{q}\right)$ and $\frac{1}{2}+n_{\sigma_{5}}\left(E_{q p}\right)$ identifiable on the last two rows of eq. (B.54) as single entities for as long as possible. They can then be combined with the real corrections, cancelling all $\lambda$-dependence, which appears at moderate values of $|p|,|q| \lesssim k_{0}$. Only the large- $q$ range requires a more careful treatment, and we return to this presently.

In order to implement this strategy, we first carry out angular integrals and substitute variables, obtaining [the virtual correction part is denoted by $\rho_{\mathcal{I}_{\mathrm{h}}}^{(\mathrm{v})}$, and $\simeq$ is a reminder of 
the divergences appearing at large $q$ and of the omission of terms of $\mathcal{O}(\epsilon)]$

$$
\begin{aligned}
\rho_{\mathcal{I}_{\mathrm{h}}}^{(\mathrm{v})} \simeq & -\frac{\pi \mathcal{K}^{2}}{(4 \pi)^{4} k} \int_{k_{-}}^{k_{+}} \mathrm{d} p \frac{n_{\sigma_{4}}\left(k_{0}-p\right) n_{\sigma_{1}}(p)}{n_{\sigma_{0}}\left(k_{0}\right)} \\
& \times\left\{\int_{-\infty}^{\infty} \frac{\mathrm{d} q}{p}\left[\frac{1}{2}+n_{\sigma_{2}}(q)\right] \ln \left|\frac{\lambda^{2}+4 p q}{\lambda^{2}}\right|\right. \\
& \left.+\left[\int_{-\infty}^{p-\lambda}+\int_{p+\lambda}^{\infty}\right] \frac{\mathrm{d} q}{p}\left|\frac{1}{2}+n_{\sigma_{5}}(q-p)\right| \ln \left|\frac{\left(\sqrt{(p-q)^{2}-\lambda^{2}}+p\right)^{2}-q^{2}}{\left(\sqrt{(p-q)^{2}-\lambda^{2}}-p\right)^{2}-q^{2}}\right|\right\} .
\end{aligned}
$$

The first "weight function" $\frac{1}{2}+n_{\sigma_{2}}(q)$ has a potential singularity at $q=0$ (if $\sigma_{2}=+1$ ), the latter at $q=p$ (if $\sigma_{5}=+1$ ); however, noticing that the combination in eq. (B.56) can be re-expressed as

$$
n_{\sigma_{4}}\left(k_{0}-p\right) n_{\sigma_{2}}(q) n_{\sigma_{5}}(p-q)=n_{\sigma_{4}}\left(k_{0}-p\right) n_{\sigma_{1}}(p)\left[\frac{1}{2}+n_{\sigma_{2}}(q)-\frac{1}{2}-n_{\sigma_{5}}(q-p)\right],
$$

these terms cancel exactly against the corresponding real corrections within the domains (e) and (f $)$ as well as (a) and (l), respectively, which are adjacent to the singular lines. An approximate form of the cancellation can be seen be rewriting eq. (B.70) in the limit $\lambda \rightarrow 0$ away from the singular lines:

$$
\begin{aligned}
\rho_{\mathcal{I}_{\mathrm{h}}}^{(\mathrm{v})} \approx & -\frac{\pi \mathcal{K}^{2}}{(4 \pi)^{4} k} \int_{k_{-}}^{k_{+}} \mathrm{d} p \frac{n_{\sigma_{4}}\left(k_{0}-p\right) n_{\sigma_{1}}(p)}{n_{\sigma_{0}}\left(k_{0}\right)} \\
& \times \int_{-\infty}^{\infty} \frac{\mathrm{d} q}{p}\left\{\left[\frac{1}{2}+n_{\sigma_{2}}(q)\right] \ln \left|\frac{4 p q}{\lambda^{2}}\right|+\left[\frac{1}{2}+n_{\sigma_{5}}(q-p)\right] \ln \left|\frac{\lambda^{2} q}{4 p(p-q)^{2}}\right|\right\} .
\end{aligned}
$$

Summing this together with eqs. (B.57)-(B.67) all $\lambda$ 's cancel, and the result is integrable in the small- $q$ domain.

It remains to deal with the ultraviolet divergence from the large- $q$ domain. The idea is to insert

$$
0=\int_{|\mathbf{q}|>\Lambda} \frac{1}{4 q^{3}}-\int_{|\mathbf{q}|>\Lambda} \frac{1}{4 q^{3}}
$$

inside the integrand representing virtual corrections. The individual terms have the same divergence as eq. (B.69):

$$
\int_{|\mathbf{q}|>\Lambda} \frac{1}{4 q^{3}}=\frac{1}{(4 \pi)^{2}}\left(\frac{1}{\epsilon}+\ln \frac{\bar{\mu}^{2}}{4 \Lambda^{2}}+2\right) .
$$

Separating the $1 / \epsilon$-part hereof, together with finite terms chosen according to the vacuum result (cf. eqs. (B.17)-(B.19) of ref. [13]), and recalling the contribution from the $\mathcal{O}(\epsilon)$-term in eq. (B.11), we obtain

$$
\begin{aligned}
\rho_{\mathcal{I}_{\mathrm{h}}}^{(\mathrm{v})}= & -\frac{\pi \mathcal{K}^{2}}{(4 \pi)^{4} k} \int_{k_{-}}^{k_{+}} \mathrm{d} p \frac{n_{\sigma_{4}}\left(k_{0}-p\right) n_{\sigma_{1}}(p)}{n_{\sigma_{0}}\left(k_{0}\right)}\left(\frac{1}{\epsilon}+2 \ln \frac{\bar{\mu}^{2}}{\mathcal{K}^{2}}+5\right) \\
& +\frac{\pi \mathcal{K}^{2}}{(4 \pi)^{4} k} \int_{k_{-}}^{k_{+}} \mathrm{d} p \frac{n_{\sigma_{4}}\left(k_{0}-p\right) n_{\sigma_{1}}(p)}{n_{\sigma_{0}}\left(k_{0}\right)} \\
& \times\left\{\ln \frac{4\left(k_{+}-p\right)\left(p-k_{-}\right) \Lambda^{2}}{k^{2} \mathcal{K}^{2}}+3+\left(\int_{-\Lambda}^{-\infty}+\int_{\Lambda}^{\infty}\right) \frac{\mathrm{d} q}{q}-(4 \pi)^{2} \int_{\mathbf{q}}[\ldots]\right\},
\end{aligned}
$$


where $[\ldots]$ refers to the original integrand from eq. (B.54). In addition it must be realized that going over to the shifted variables of eq. (B.70) has introduced an "error" (because we have carelessly handled logarithmically divergent integrals) which must now be compensated for. Indeed, eq. (B.74) and an infrared (IR) part from $|q|<\Lambda$ only add up to the correct eq. (B.69) if the IR part yields

$$
\frac{1}{(4 \pi)^{2}}\left(\frac{4 \Lambda^{2}}{\lambda^{2}}-1\right)
$$

Yet the corresponding contribution from the vacuum parts of eq. (B.72) reads

$$
\frac{1}{(4 \pi)^{2}} \int_{-\Lambda}^{\Lambda} \frac{\mathrm{d} q}{2 p}\left\{\operatorname{sign}(q) \ln \left|\frac{4 p q}{\lambda^{2}}\right|+\operatorname{sign}(q-p) \ln \left|\frac{\lambda^{2} q}{4 p(p-q)^{2}}\right|\right\}=\frac{1}{(4 \pi)^{2}}\left(\frac{4 \Lambda^{2}}{\lambda^{2}}+1\right) .
$$

The difference of eqs. (B.76) and (B.77) needs to be cancelled from the integrand of eq. (B.75) if we use the shifted variables. Thereby the final expression reads

$$
\begin{aligned}
\rho_{\mathcal{I}_{\mathrm{h}}}^{(\mathrm{v})}=\rho_{\mathcal{I}_{\mathrm{h}}}^{\mathrm{vac}} & +\frac{\pi \mathcal{K}^{2}}{(4 \pi)^{4} k} \int_{k_{-}}^{k_{+}} \mathrm{d} p \frac{n_{\sigma_{4}}\left(k_{0}-p\right) n_{\sigma_{1}}(p)}{n_{\sigma_{0}}\left(k_{0}\right)} \\
& \times\left\{\ln \frac{4\left(k_{+}-p\right)\left(p-k_{-}\right) \Lambda^{2}}{k^{2} \mathcal{K}^{2}}+5+\int_{-\infty}^{\infty} \frac{\mathrm{d} q}{p}\left[\frac{p \theta(|q|-\Lambda)}{|q|}\right.\right. \\
& \left.\left.+\left(\frac{1}{2}+n_{\sigma_{2}}(q)\right) \ln \left|\frac{\lambda^{2}}{4 p q}\right|-\left(\frac{1}{2}+n_{\sigma_{5}}(q-p)\right) \ln \left|\frac{\lambda^{2} q}{4 p(p-q)^{2}}\right|\right]\right\}
\end{aligned}
$$

where a vacuum part has been defined as

$$
\rho_{\mathcal{I}_{\mathrm{h}}}^{\mathrm{vac}} \equiv-\frac{\pi \mathcal{K}^{2} T}{(4 \pi)^{4} k} \ln \left(\frac{e^{k_{+} / T}+\sigma_{0} e^{-k_{+} / T}-\sigma_{1}-\sigma_{4}}{e^{k_{-} / T}+\sigma_{0} e^{-k_{-} / T}-\sigma_{1}-\sigma_{4}}\right)\left(\frac{1}{\epsilon}+2 \ln \frac{\bar{\mu}^{2}}{\mathcal{K}^{2}}+5\right) .
$$

There is no dependence on $\Lambda$ in eq. (B.78), and the $1 / q$-tails at $|q|>\Lambda$ cancel as well so that, when combined with the real corrections, the expression is integrable.

For $k_{+}, k_{-} \gg \pi T$, the ultraviolet asymptotics of $\rho_{\mathcal{I}_{\mathrm{h}}}$ reads [13]

$$
\begin{aligned}
\rho_{\mathcal{I}_{\mathrm{h}}}{ }^{k_{+}, k_{-} \gg \pi T} & -\frac{\mathcal{K}^{2}}{4(4 \pi)^{3}}\left(\frac{1}{\epsilon}+2 \ln \frac{\bar{\mu}^{2}}{\mathcal{K}^{2}}+5\right) \\
& +\int_{\mathbf{p}}\left\{\frac{n_{\sigma_{4}}-\left(n_{\sigma_{2}}+n_{\sigma_{5}}\right)}{16 \pi p}+\frac{p\left[3 n_{\sigma_{4}}-\left(n_{\sigma_{2}}+n_{\sigma_{5}}\right)\right]}{12 \pi} \frac{k_{0}^{2}+\frac{k^{2}}{3}}{\mathcal{K}^{4}}\right\} .
\end{aligned}
$$

The specific statistics needed in the present case are

$$
\begin{aligned}
& \mathcal{I}_{\mathrm{h}} \Leftrightarrow\left(\sigma_{1} \sigma_{4} \sigma_{5} \mid \sigma_{2} \sigma_{0}\right)=(+-+\mid+-), \\
& \widetilde{\mathcal{I}}_{\mathrm{h}} \Leftrightarrow\left(\sigma_{1} \sigma_{4} \sigma_{5} \mid \sigma_{2} \sigma_{0}\right)=(+--\mid--), \\
& \widehat{\mathcal{I}}_{\mathrm{h}} \Leftrightarrow\left(\sigma_{1} \sigma_{4} \sigma_{5} \mid \sigma_{2} \sigma_{0}\right)=(-+-\mid+-),
\end{aligned}
$$

where only the first three indices are independent. For numerical evaluation, we have reflected the final integral to the domain defined in figure 6 of ref. [15]. The corresponding integrand is not shown explicitly, since no substantial cancellations take place in the reflection. Results of numerical evaluations (after subtracting the vacuum part, cf. eq. (B.79)) are shown in figure 10, and are seen to agree with the OPE-asymptotics from eq. (B.80) as well as with the $k \rightarrow 0$ limit from ref. [24]. 

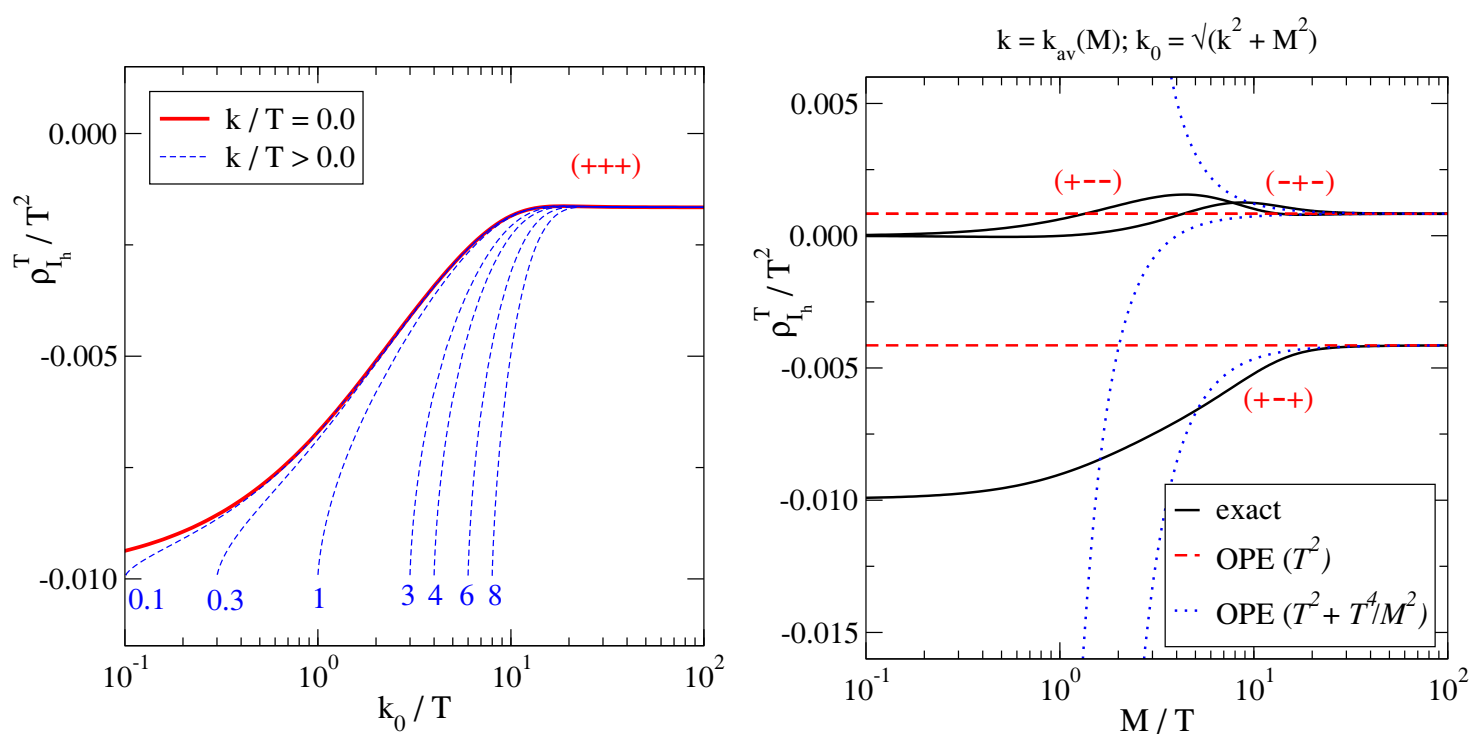

Figure 10. Left: the thermal part of $\rho_{\mathcal{I}_{\mathrm{h}}}$ with the purely bosonic statistics $\left(\sigma_{1} \sigma_{4} \sigma_{5}\right)=(+++)$, for $k_{0} \geq k+0.001 T$, compared with the zero-momentum limit determined in ref. [24]. Right: the thermal part of $\rho_{\mathcal{I}_{\mathrm{h}}}$ with the momentum of eq. (B.19) and statistics of eqs. (B.81)-(B.83) as a function of $M / T$, compared with the OPE-asymptotics from eq. (B.80).

\section{B.10 $\rho_{\mathcal{I}_{\mathrm{h}}}$,}

After carrying out the Matsubara sums for $\mathcal{I}_{\mathrm{h}}$, we get

$$
\begin{aligned}
& \mathcal{I}_{\mathrm{h}},=\lim _{\lambda \rightarrow 0} \int_{\mathbf{p}, \mathbf{q}} \frac{2}{8 \epsilon_{q} \epsilon_{p k} E_{q p}}\{ \\
& \frac{-i k_{n} \epsilon_{q}+\mathbf{k} \cdot \mathbf{q}}{-i k_{n}+\epsilon_{p k}+\epsilon_{q}+E_{q p}} \frac{\left[1+n_{\sigma_{4}}\left(\epsilon_{p k}\right)+n_{\sigma_{2}}\left(\epsilon_{q}\right)\right]\left[1+n_{\sigma_{5}}\left(E_{q p}\right)\right]+n_{\sigma_{4}}\left(\epsilon_{p k}\right) n_{\sigma_{2}}\left(\epsilon_{q}\right)}{\epsilon_{p}^{2}-\left(\epsilon_{q}+E_{q p}\right)^{2}} \\
& +\frac{-i k_{n} \epsilon_{q}+\mathbf{k} \cdot \mathbf{q}}{-i k_{n}-\epsilon_{p k}+\epsilon_{q}+E_{q p}} \frac{n_{\sigma_{4}}\left(\epsilon_{p k}\right)\left[1+n_{\sigma_{2}}\left(\epsilon_{q}\right)+n_{\sigma_{5}}\left(E_{q p}\right)\right]-n_{\sigma_{2}}\left(\epsilon_{q}\right) n_{\sigma_{5}}\left(E_{q p}\right)}{\epsilon_{p}^{2}-\left(\epsilon_{q}+E_{q p}\right)^{2}} \\
& +\frac{+i k_{n} \epsilon_{q}+\mathbf{k} \cdot \mathbf{q}}{-i k_{n}+\epsilon_{p k}-\epsilon_{q}+E_{q p}} \frac{n_{\sigma_{2}}\left(\epsilon_{q}\right)\left[1+n_{\sigma_{4}}\left(\epsilon_{p k}\right)+n_{\sigma_{5}}\left(E_{q p}\right)\right]-n_{\sigma_{4}}\left(\epsilon_{p k}\right) n_{\sigma_{5}}\left(E_{q p}\right)}{\epsilon_{p}^{2}-\left(\epsilon_{q}-E_{q p}\right)^{2}} \\
& \left.+\frac{-i k_{n} \epsilon_{q}+\mathbf{k} \cdot \mathbf{q}}{-i k_{n}+\epsilon_{p k}+\epsilon_{q}-E_{q p}} \frac{n_{\sigma_{5}}\left(E_{q p}\right)\left[1+n_{\sigma_{4}}\left(\epsilon_{p k}\right)+n_{\sigma_{2}}\left(\epsilon_{q}\right)\right]-n_{\sigma_{4}}\left(\epsilon_{p k}\right) n_{\sigma_{2}}\left(\epsilon_{q}\right)}{\epsilon_{p}^{2}-\left(\epsilon_{q}-E_{q p}\right)^{2}}\right\} \\
& +\lim _{\lambda \rightarrow 0} \int_{\mathbf{p}} \frac{2}{4 \epsilon_{p} \epsilon_{p k}}\left[\frac{1+n_{\sigma_{1}}\left(\epsilon_{p}\right)+n_{\sigma_{4}}\left(\epsilon_{p k}\right)}{-i k_{n}+\epsilon_{p}+\epsilon_{p k}}+\frac{n_{\sigma_{1}}\left(\epsilon_{p}\right)-n_{\sigma_{4}}\left(\epsilon_{p k}\right)}{i k_{n}-\epsilon_{p}+\epsilon_{p k}}\right] \\
& \times \int_{\mathbf{q}}\left[\frac{1+n_{\sigma_{2}}\left(\epsilon_{q}\right)+n_{\sigma_{5}}\left(E_{q p}\right)}{4 \epsilon_{q} E_{q p}}\left(\frac{i k_{n} \epsilon_{q}+\mathbf{k} \cdot \mathbf{q}}{\epsilon_{p}+\epsilon_{q}+E_{q p}}-\frac{-i k_{n} \epsilon_{q}+\mathbf{k} \cdot \mathbf{q}}{\epsilon_{p}-\epsilon_{q}-E_{q p}}\right)\right. \\
& \left.+\frac{n_{\sigma_{2}}\left(\epsilon_{q}\right)-n_{\sigma_{5}}\left(E_{q p}\right)}{4 \epsilon_{q} E_{q p}}\left(\frac{-i k_{n} \epsilon_{q}+\mathbf{k} \cdot \mathbf{q}}{\epsilon_{p}-\epsilon_{q}+E_{q p}}-\frac{i k_{n} \epsilon_{q}+\mathbf{k} \cdot \mathbf{q}}{\epsilon_{p}+\epsilon_{q}-E_{q p}}\right)\right] \\
& +\left(i k_{n} \rightarrow-i k_{n}\right) \text {. }
\end{aligned}
$$


Taking the cut like in eq. (A.27), the first four structures are real corrections, the last two are virtual corrections. The corresponding scattering processes can be depicted like in figure 3 of ref. [15], with internal propagators carrying the index $\sigma_{3}$ shrunk to points.

For the real corrections, eq. (4.28) of ref. [15] now reads

$$
\begin{aligned}
\left\langle\Phi_{\mathrm{r} 1}\left(k_{0}-p_{0}|q| p_{0}-q \mid \cdot\right)\right\rangle & =-\left\langle\Phi_{\mathrm{r} 2}\left(p_{0}-k_{0}|q| p_{0}-q \mid \cdot\right)\right\rangle \\
& =-\left\langle\Phi_{\mathrm{r} 3}\left(k_{0}-p_{0}|-q| p_{0}-q \mid \cdot\right)\right\rangle \\
& =-\left\langle\Phi_{\mathrm{r} 4}\left(k_{0}-p_{0}|q| q-p_{0} \mid \cdot\right)\right\rangle \\
& =\frac{n_{\sigma_{4}}\left(k_{0}-p_{0}\right) n_{\sigma_{2}}(q) n_{\sigma_{5}}\left(p_{0}-q\right)}{n_{\sigma_{0}}\left(k_{0}\right)} \times \mathbb{P}\left\langle\frac{k_{0} q-\mathbf{k} \cdot \mathbf{q}}{p_{0}^{2}-p^{2}}\right\rangle_{\left(k_{0}-p_{0}|q| p_{0}-q \mid \cdot\right)}
\end{aligned}
$$

where the arguments $(\ldots|\ldots| \ldots \mid \cdot)$ on the last line refer to $\epsilon_{p k}, \epsilon_{q}$, and $E_{q p}$, respectively. The azimuthal average, as defined in ref. [15], yields

$$
\langle\mathbf{k} \cdot \mathbf{q}\rangle_{\left(\epsilon_{p k}|q| E_{q p}\right)}=\frac{\left(p^{2}+k^{2}-\epsilon_{p k}^{2}\right)\left(p^{2}+q^{2}+\lambda^{2}-E_{q p}^{2}\right)}{4 p^{2}}
$$

The subsequent $p$-integral leads to logarithms and fractions. Renaming finally $p_{0} \rightarrow p$ and factoring out

$$
\frac{\pi n_{\sigma_{4}}\left(k_{0}-p\right) n_{\sigma_{2}}(q) n_{\sigma_{5}}(p-q)}{(4 \pi)^{4} k n_{\sigma_{0}}\left(k_{0}\right)}
$$

the $\lambda \rightarrow 0$ limits (in the sense specified in ref. [15]) read:

$$
\begin{aligned}
& (\mathrm{a})=(\mathrm{l}): 2(p-q)\left[1+\frac{\left(p-2 k_{-}\right)\left(p-2 k_{+}\right)}{p^{2}}\right]+\frac{q \mathcal{K}^{2}}{p^{2}} \ln \left|\frac{4 p(p-q)^{2}}{\lambda^{2} q}\right|, \\
& \text { (b) : } \frac{4 k_{-}\left(k_{+}-q\right)}{p}+\frac{q \mathcal{K}^{2}}{p^{2}} \ln \left|\frac{k_{+}(p-q)}{q\left(p-k_{+}\right)}\right| \text {, } \\
& (\underline{\mathrm{b}}): 2\left(p-k_{-}\right)\left[1+\frac{(p-2 q)\left(p-2 k_{+}\right)}{p^{2}}\right]+\frac{q \mathcal{K}^{2}}{p^{2}} \ln \left|\frac{4 p(p-q)\left(p-k_{-}\right)}{\lambda^{2} k_{-}}\right| \text {, } \\
& (\mathrm{c})=-(\mathrm{h})=-(\underline{\mathrm{h}})=-(\mathrm{j}): \quad \frac{4 q\left(k_{+}-k_{-}\right)}{p}+\frac{q \mathcal{K}^{2}}{p^{2}} \ln \left|\frac{k_{+}\left(p-k_{-}\right)}{k_{-}\left(p-k_{+}\right)}\right|, \\
& (\tilde{\mathrm{c}}): \quad 2 k_{-}\left[1-\frac{(p-2 q)\left(p-2 k_{+}\right)}{p^{2}}\right]+\frac{q \mathcal{K}^{2}}{p^{2}} \ln \left|\frac{4 k_{-} p(p-q)}{\lambda^{2}\left(p-k_{-}\right)}\right|, \\
& \text {(d): } \frac{4\left(p-k_{-}\right)\left(k_{+}-p+q\right)}{p}+\frac{q \mathcal{K}^{2}}{p^{2}} \ln \left|\frac{q k_{+}}{(p-q)\left(p-k_{+}\right)}\right| \text {, } \\
& (\mathrm{e})=(\mathrm{f}): \quad \frac{4(p-q)\left(k_{+}+k_{-}-p\right)}{p}+\frac{q \mathcal{K}^{2}}{p^{2}} \ln \left|\frac{k_{+} k_{-}}{\left(p-k_{+}\right)\left(p-k_{-}\right)}\right| \text {, } \\
& (\underline{\mathrm{e}})=(\underline{\mathrm{f}}): \quad 2 q\left[1-\frac{\left(p-2 k_{-}\right)\left(p-2 k_{+}\right)}{p^{2}}\right]+\frac{q \mathcal{K}^{2}}{p^{2}} \ln \left|\frac{4 p q}{\lambda^{2}}\right|,
\end{aligned}
$$




$$
\begin{aligned}
& (\mathrm{g})=(\underline{\mathrm{g}}): \quad \frac{4\left(p-k_{+}\right)\left(k_{-}-p+q\right)}{p}+\frac{q \mathcal{K}^{2}}{p^{2}} \ln \left|\frac{q k_{-}}{(p-q)\left(p-k_{-}\right)}\right|, \\
& (\mathrm{i})=(\underline{\mathrm{k}}): \quad \frac{4 k_{+}\left(k_{-}-q\right)}{p}+\frac{q \mathcal{K}^{2}}{p^{2}} \ln \left|\frac{k_{-}(p-q)}{q\left(p-k_{-}\right)}\right|, \\
& (\underline{\mathrm{i}})=(\mathrm{k}): \quad 2\left(p-k_{+}\right)\left[1+\frac{(p-2 q)\left(p-2 k_{-}\right)}{p^{2}}\right]+\frac{q \mathcal{K}^{2}}{p^{2}} \ln \left|\frac{4 p(p-q)\left(p-k_{+}\right)}{\lambda^{2} k_{+}}\right| .
\end{aligned}
$$

As far as the virtual corrections go, they include a divergent part:

$$
\begin{aligned}
\rho_{\mathcal{I}_{\mathrm{h}}}, \ni & \int_{\mathbf{p}} \frac{2 \pi \delta\left(k_{0}-\epsilon_{p}-\epsilon_{p k}\right) n_{\sigma_{4}}\left(k_{0}-p\right) n_{\sigma_{1}}(p)}{4 \epsilon_{p} \epsilon_{p k} n_{\sigma_{0}}\left(k_{0}\right)} \\
& \times \int_{\mathbf{q}} \mathbb{P}\left\{\frac{1}{4 \epsilon_{q} E_{q p}}\left(\frac{k_{0} \epsilon_{q}+\mathbf{k} \cdot \mathbf{q}}{\epsilon_{p}+\epsilon_{q}+E_{q p}}+\frac{-k_{0} \epsilon_{q}+\mathbf{k} \cdot \mathbf{q}}{-\epsilon_{p}+\epsilon_{q}+E_{q p}}\right)\right\} \\
= & -\frac{2 \rho_{\mathcal{J}_{\mathrm{b}}}}{\mathcal{K}^{2}} \times\left.\operatorname{Re} \int_{Q} \frac{K \cdot Q}{Q^{2}\left[(Q-P)^{2}+\lambda^{2}\right]}\right|_{k_{n}=-i k_{0}, p_{n}=-i \epsilon_{p}, \epsilon_{p k}=k_{0}-\epsilon_{p}} .
\end{aligned}
$$

Here we made use of the fact that the vacuum integral is independent of $p$ :

$$
\left.\operatorname{Re} \int_{Q} \frac{K \cdot Q}{Q^{2}\left[(Q-P)^{2}+\lambda^{2}\right]}\right|_{k_{n}=-i k_{0}, p_{n}=-i \epsilon_{p}, \epsilon_{p k}=k_{0}-\epsilon_{p}}=-\frac{\mathcal{K}^{2}}{4(4 \pi)^{2}}\left(\frac{1}{\epsilon}+\ln \frac{\bar{\mu}^{2}}{\lambda^{2}}+\frac{1}{2}\right) .
$$

Like with $\rho_{\mathcal{I}_{\mathrm{h}}}$ it is helpful, however, not to separate the vacuum integral from the outset, but rather to treat the structures $\frac{1}{2}+n_{\sigma_{2}}\left(\epsilon_{q}\right)$ and $\frac{1}{2}+n_{\sigma_{5}}\left(E_{q p}\right)$ identifiable on the last two rows of eq. (B.84) as single entities for as long as possible.

In order to implement this, we first carry out angular integrals and substitute integration variables, obtaining [the virtual correction part is denoted by $\rho_{\mathcal{I}_{\mathrm{h}}}^{(\mathrm{v})}$, and $\simeq$ is a reminder of the divergences appearing at large $q$ and of the omission of terms of $\mathcal{O}(\epsilon)$ ]

$$
\begin{aligned}
\rho_{\mathcal{I}_{\mathrm{h}}}^{(\mathrm{v})} \simeq & \frac{\pi}{(4 \pi)^{4} k} \int_{k_{-}}^{k_{+}} \mathrm{d} p \frac{n_{\sigma_{4}}\left(k_{0}-p\right) n_{\sigma_{1}}(p)}{n_{\sigma_{0}}\left(k_{0}\right)} \\
& \times\left\{\int_{-\infty}^{\infty} \mathrm{d} q\left[\frac{1}{2}+n_{\sigma_{2}}(q)\right]\left[\frac{2 q\left(\mathcal{K}^{2}-2 p k_{0}\right)}{p^{2}}+\frac{2 q p \mathcal{K}^{2}+\lambda^{2}\left(\mathcal{K}^{2}-2 p k_{0}\right)}{2 p^{3}} \ln \left|\frac{\lambda^{2}}{\lambda^{2}+4 p q}\right|\right]\right. \\
& -\left[\int_{-\infty}^{p-\lambda}+\int_{p+\lambda}^{\infty}\right] \mathrm{d} q\left|\frac{1}{2}+n_{\sigma_{5}}(q-p)\right|\left[\frac{2\left(\mathcal{K}^{2}-2 p k_{0}\right) \sqrt{(q-p)^{2}-\lambda^{2}}}{p^{2}}\right. \\
& \left.\left.+\frac{2 q p \mathcal{K}^{2}+\lambda^{2}\left(\mathcal{K}^{2}-2 p k_{0}\right)}{2 p^{3}} \ln \left|\frac{\left(\sqrt{(p-q)^{2}-\lambda^{2}}+p\right)^{2}-q^{2}}{\left(\sqrt{(p-q)^{2}-\lambda^{2}}-p\right)^{2}-q^{2}}\right|\right]\right\} .
\end{aligned}
$$

The first "weight function" $\frac{1}{2}+n_{\sigma_{2}}(q)$ has a potential singularity at $q=0$ (if $\sigma_{2}=+1$ ), the latter at $q=p$ (if $\sigma_{5}=+1$ ); however, making use of eq. (B.71), it can be seen that these terms cancel (apart from a harmless $\sim \sqrt{(q-p)^{2}-\lambda^{2}}$ in the latter case) against the corresponding real corrections within the domains ( $\underline{\mathrm{e}})$ and $(\underline{\mathrm{f}})$ as well as (a) and (l), respectively, which are adjacent to the singular lines. An approximate form of the cancellation 
can be seen be rewriting eq. (B.101) in the limit $\lambda \rightarrow 0$ away from the singular lines:

$$
\begin{aligned}
\rho_{\mathcal{I}_{\mathrm{h}}}^{(\mathrm{v})} \approx & \frac{\pi}{(4 \pi)^{4} k} \int_{k_{-}}^{k_{+}} \mathrm{d} p \frac{n_{\sigma_{4}}\left(k_{0}-p\right) n_{\sigma_{1}}(p)}{n_{\sigma_{0}}\left(k_{0}\right)} \\
& \times \int_{-\infty}^{\infty} \mathrm{d} q\left\{\left[\frac{1}{2}+n_{\sigma_{2}}(q)\right]\left[\frac{2 q\left(\mathcal{K}^{2}-2 p k_{0}\right)}{p^{2}}+\frac{q \mathcal{K}^{2}}{p^{2}} \ln \left|\frac{\lambda^{2}}{4 p q}\right|\right]\right. \\
& \left.-\left[\frac{1}{2}+n_{\sigma_{5}}(q-p)\right]\left[\frac{2(q-p)\left(\mathcal{K}^{2}-2 p k_{0}\right)}{p^{2}}+\frac{q \mathcal{K}^{2}}{p^{2}} \ln \left|\frac{\lambda^{2} q}{4 p(p-q)^{2}}\right|\right]\right\} .
\end{aligned}
$$

Summing together with eqs. (B.88)-(B.98), all $\lambda$ 's cancel, and the remainder is integrable in the IR domain $|p|,|q| \lesssim k_{0}$.

It remains to deal with the ultraviolet divergence. We add

$$
0=-\frac{\mathcal{K}^{2}}{4} \int_{|\mathbf{q}|>\Lambda} \frac{1}{4 q^{3}}+\frac{\mathcal{K}^{2}}{4} \int_{|\mathbf{q}|>\Lambda} \frac{1}{4 q^{3}}
$$

in the integrand of the virtual corrections. As seen from eq. (B.74) the individual terms have the same divergence as eq. (B.100). Separating the 1/ $\epsilon$-part hereof, together with finite terms chosen according to the vacuum result (cf. eq. (B.20) of ref. [13]), and recalling the contribution from the $\mathcal{O}(\epsilon)$-term in eq. (B.11), we obtain

$$
\begin{aligned}
\rho_{\mathcal{I}_{\mathrm{h}}}^{(\mathrm{v})}= & -\frac{\pi \mathcal{K}^{2}}{2(4 \pi)^{4} k} \int_{k_{-}}^{k_{+}} \mathrm{d} p \frac{n_{\sigma_{4}}\left(k_{0}-p\right) n_{\sigma_{1}}(p)}{n_{\sigma_{0}}\left(k_{0}\right)}\left(\frac{1}{\epsilon}+2 \ln \frac{\bar{\mu}^{2}}{\mathcal{K}^{2}}+\frac{9}{2}\right) \\
& +\frac{\pi \mathcal{K}^{2}}{2(4 \pi)^{4} k} \int_{k_{-}}^{k_{+}} \mathrm{d} p \frac{n_{\sigma_{4}}\left(k_{0}-p\right) n_{\sigma_{1}}(p)}{n_{\sigma_{0}}\left(k_{0}\right)} \\
& \times\left\{\ln \frac{4\left(k_{+}-p\right)\left(p-k_{-}\right) \Lambda^{2}}{k^{2} \mathcal{K}^{2}}+\frac{5}{2}+\left(\int_{-\Lambda}^{-\infty}+\int_{\Lambda}^{\infty}\right) \frac{\mathrm{d} q}{q}+\frac{4(4 \pi)^{2}}{\mathcal{K}^{2}} \int_{\mathbf{q}}[\ldots]\right\},
\end{aligned}
$$

where $[\ldots]$ refers to original integrand in eq. (B.84). In addition it must be realized that going over to the shifted variables of eq. (B.101) has introduced an "error" (because we have carelessly handled logarithmically divergent integrals) which must now be compensated for. In order for the ultraviolet contribution $-\frac{\mathcal{K}^{2}}{4} \int_{|\mathbf{q}|>\Lambda} \frac{1}{4 q^{3}}$ (cf. eq. (B.74)) and the infrared contribution from $|q|<\Lambda$ to add up to the correct result in eq. (B.100), the vacuum terms of the latter should yield

$$
-\frac{\mathcal{K}^{2}}{4(4 \pi)^{2}}\left(\frac{4 \Lambda^{2}}{\lambda^{2}}-\frac{3}{2}\right)
$$

Explicit integration shows, however, that they yield

$$
\begin{aligned}
& \frac{1}{2(4 \pi)^{2}} \int_{-\Lambda}^{\Lambda} \mathrm{d} q\left\{\frac{\operatorname{sign}(q)}{2}\left[\frac{2 q\left(\mathcal{K}^{2}-2 p k_{0}\right)}{p^{2}}+\frac{q \mathcal{K}^{2}}{p^{2}} \ln \left|\frac{\lambda^{2}}{4 p q}\right|\right]\right. \\
& \left.-\frac{\operatorname{sign}(q-p)}{2}\left[\frac{2(q-p)\left(\mathcal{K}^{2}-2 p k_{0}\right)}{p^{2}}+\frac{q \mathcal{K}^{2}}{p^{2}} \ln \left|\frac{\lambda^{2} q}{4 p(p-q)^{2}}\right|\right]\right\} \\
& =\frac{1}{(4 \pi)^{2}}\left[k_{0} p-\frac{\mathcal{K}^{2}}{4}\left(\ln \frac{4 \Lambda^{2}}{\lambda^{2}}+\frac{1}{2}\right)\right] .
\end{aligned}
$$


The difference of eqs. (B.106) and (B.105) needs to be cancelled from the integrand of eq. (B.104) if we employ the shifted variables. This finally yields

$$
\begin{aligned}
\rho_{\mathcal{I}_{\mathrm{h}}}^{(\mathrm{v})}=\rho_{\mathcal{I}_{\mathrm{h}}}^{\mathrm{vac}} & +\frac{\pi \mathcal{K}^{2}}{2(4 \pi)^{4} k} \int_{k_{-}}^{k_{+}} \mathrm{d} p \frac{n_{\sigma_{4}}\left(k_{0}-p\right) n_{\sigma_{1}}(p)}{n_{\sigma_{0}}\left(k_{0}\right)} \\
\times\{\ln & \frac{4\left(k_{+}-p\right)\left(p-k_{-}\right) \Lambda^{2}}{k^{2} \mathcal{K}^{2}}+\frac{9}{2}-\frac{4 k_{0} p}{\mathcal{K}^{2}}+\int_{-\infty}^{\infty} \mathrm{d} q\left[\frac{\theta(|q|-\Lambda)}{|q|}\right. \\
& +\left(\frac{1}{2}+n_{\sigma_{2}}(q)\right)\left(\frac{4 q\left(\mathcal{K}^{2}-2 p k_{0}\right)}{p^{2} \mathcal{K}^{2}}+\frac{2 q}{p^{2}} \ln \left|\frac{\lambda^{2}}{4 p q}\right|\right) \\
& \left.\left.-\left(\frac{1}{2}+n_{\sigma_{5}}(q-p)\right)\left(\frac{4(q-p)\left(\mathcal{K}^{2}-2 p k_{0}\right)}{p^{2} \mathcal{K}^{2}}+\frac{2 q}{p^{2}} \ln \left|\frac{\lambda^{2} q}{4 p(p-q)^{2}}\right|\right)\right]\right\},
\end{aligned}
$$

where the vacuum part has been defined as

$$
\rho_{\mathcal{I}_{\mathrm{h}}}^{\mathrm{vac}} \equiv-\frac{\pi \mathcal{K}^{2} T}{2(4 \pi)^{4} k} \ln \left(\frac{e^{k_{+} / T}+\sigma_{0} e^{-k_{+} / T}-\sigma_{1}-\sigma_{4}}{e^{k_{-} / T}+\sigma_{0} e^{-k_{-} / T}-\sigma_{1}-\sigma_{4}}\right)\left(\frac{1}{\epsilon}+2 \ln \frac{\bar{\mu}^{2}}{\mathcal{K}^{2}}+\frac{9}{2}\right) .
$$

There is no dependence on $\Lambda$ in eq. (B.107), and the $1 / q$-tails at $|q|>\Lambda$ cancel as well, so that the expression is integrable once combined with the real corrections. (In fact the integrand also has a constant part at large $|q|$, but this cancels due to its antisymmetry in $q \rightarrow-q$.

For $k_{+}, k_{-} \gg \pi T$, the ultraviolet asymptotics of the spectral function reads [13]

$$
\begin{aligned}
\rho_{\mathcal{I}_{\mathrm{h}},}{ }^{k_{+}, k_{-} \gg \pi T} & -\frac{\mathcal{K}^{2}}{8(4 \pi)^{3}}\left(\frac{1}{\epsilon}+2 \ln \frac{\bar{\mu}^{2}}{\mathcal{K}^{2}}+\frac{9}{2}\right) \\
& +\int_{\mathbf{p}}\left\{\frac{n_{\sigma_{4}}}{16 \pi p}+\frac{p\left[3\left(n_{\sigma_{4}}-n_{\sigma_{2}}\right)+n_{\sigma_{5}}\right]}{24 \pi} \frac{k_{0}^{2}+\frac{k^{2}}{3}}{\mathcal{K}^{4}}\right\} .
\end{aligned}
$$

The specific statistics needed in this paper are

$$
\widehat{\mathcal{I}}_{\mathrm{h}}, \Leftrightarrow\left(\sigma_{1} \sigma_{4} \sigma_{5} \mid \sigma_{2} \sigma_{0}\right)=(-+-\mid+-) .
$$

For numerical evaluation, we have reflected the final integral to the domain defined in figure 6 of ref. [15]. The corresponding integrand is not shown explicitly, since no substantial cancellations take place in the reflection. Results of numerical evaluations are shown in figure 11, and it can be seen that the OPE-asymptotics from eq. (B.109) as well as the $k \rightarrow 0$ limit from ref. [24] are reproduced. For $\sigma_{2}=\sigma_{5}$ we have also checked that the identity $\rho_{\mathcal{I}_{\mathrm{h}}}=\frac{1}{2}\left(\rho_{\mathcal{I}_{\mathrm{f}}}+\rho_{\mathcal{I}_{\mathrm{h}}}\right)$, obtained by substitutions of sum-integration variables, is satisfied.

\section{Choice of parameters}

For illustration we choose $M=10^{7} \mathrm{GeV}$ for the numerics like in refs. [7, 8]. The physical Higgs mass is set to $m_{H}=126 \mathrm{GeV}$. In order to convert pole masses and the muon decay constant to $\overline{\mathrm{MS}}$ scheme parameters at a scale $\bar{\mu}=\bar{\mu}_{0} \equiv m_{Z}$ we employ 1-loop relations specified in ref. [26]; subsequently, 1-loop renormalization group equations determine the running of the couplings to a scale

$$
\bar{\mu}_{\text {ref }} \equiv \max (M, \pi T) .
$$



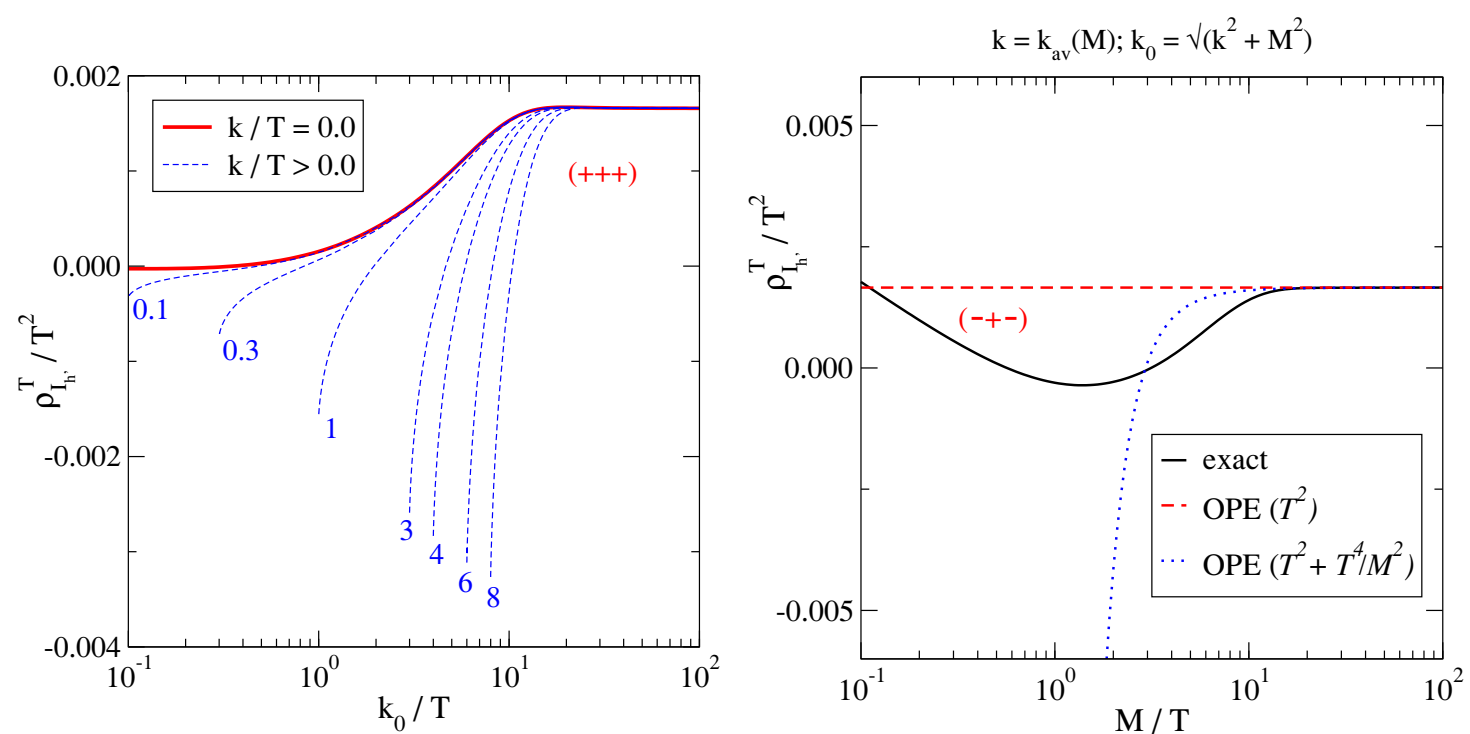

Figure 11. Left: the thermal part of $\rho_{\mathcal{I}_{\mathrm{h}}}$, with the purely bosonic statistics $\left(\sigma_{1} \sigma_{4} \sigma_{5}\right)=(+++)$, for $k_{0} \geq k+0.001 T$, compared with the zero-momentum limit determined in ref. [24]. Right: the thermal part of $\rho_{\mathcal{I}_{\mathrm{h}}}$, with the momentum of eq. (B.19) and statistics of eq. (B.110) as a function of $M / T$, compared with the OPE-asymptotics from eq. (B.109).

Within this approximation the $\mathrm{U}(1), \mathrm{SU}(2)$ and $\mathrm{SU}(3)$ gauge couplings $g_{1}^{2}, g_{2}^{2}, g_{3}^{2}$ have explicit solutions (we have set $N_{\mathrm{c}}=3$ and considered 3 families),

$$
g_{1}^{2}(\bar{\mu})=\frac{48 \pi^{2}}{41 \ln \left(\Lambda_{1} / \bar{\mu}\right)}, \quad g_{2}^{2}(\bar{\mu})=\frac{48 \pi^{2}}{19 \ln \left(\bar{\mu} / \Lambda_{2}\right)}, \quad g_{3}^{2}(\bar{\mu})=\frac{24 \pi^{2}}{21 \ln \left(\bar{\mu} / \Lambda_{3}\right)},
$$

where $\Lambda_{1}, \Lambda_{2}, \Lambda_{3}$ are solved from the boundary values at $\bar{\mu}=\bar{\mu}_{0}$. The top Yukawa and the Higgs self-coupling at $\bar{\mu}>\bar{\mu}_{0}$ are solved numerically from

$$
\begin{aligned}
\bar{\mu} \frac{\mathrm{d} h_{t}^{2}}{\mathrm{~d} \bar{\mu}} & =\frac{h_{t}^{2}}{8 \pi^{2}}\left[\frac{9}{2} h_{t}^{2}-\frac{17}{12} g_{1}^{2}-\frac{9}{4} g_{2}^{2}-8 g_{3}^{2}\right], \\
\bar{\mu} \frac{\mathrm{d} \lambda}{\mathrm{d} \bar{\mu}} & =\frac{1}{8 \pi^{2}}\left[\frac{3}{16}\left(g_{1}^{4}+2 g_{1}^{2} g_{2}^{2}+3 g_{2}^{4}\right)-\frac{3}{2} \lambda\left(g_{1}^{2}+3 g_{2}^{2}\right)+12 \lambda^{2}+6 \lambda h_{t}^{2}-3 h_{t}^{4}\right] .
\end{aligned}
$$

Given many confusions in leptogenesis literature concerning the physical value of $\lambda$ (numbers too large by a factor of 2 or 4 can be found even though everyone uses the same eq. (3.7)), let us recall that at tree level $\lambda \approx g_{2}^{2} m_{H}^{2} /\left(8 m_{W}^{2}\right) \approx 0.13$.

\section{References}

[1] M. Fukugita and T. Yanagida, Baryogenesis Without Grand Unification, Phys. Lett. B 174 (1986) 45 [INSPIRE].

[2] W. Buchmüller, R. Peccei and T. Yanagida, Leptogenesis as the origin of matter, Ann. Rev. Nucl. Part. Sci. 55 (2005) 311 [hep-ph/0502169] [InSPIRE].

[3] S. Davidson, E. Nardi and Y. Nir, Leptogenesis, Phys. Rept. 466 (2008) 105 [arXiv:0802.2962] [INSPIRE]. 
[4] X.-D. Shi and G.M. Fuller, A new dark matter candidate: nonthermal sterile neutrinos, Phys. Rev. Lett. 82 (1999) 2832 [astro-ph/9810076] [INSPIRE].

[5] L. Canetti, M. Drewes, T. Frossard and M. Shaposhnikov, Dark Matter, Baryogenesis and Neutrino Oscillations from Right Handed Neutrinos, Phys. Rev. D 87 (2013) 093006 [arXiv: 1208.4607] [INSPIRE].

[6] D. Besak and D. Bödeker, Hard thermal loops for soft or collinear external momenta, JHEP 05 (2010) 007 [arXiv: 1002.0022] [INSPIRE].

[7] A. Anisimov, D. Besak and D. Bödeker, Thermal production of relativistic Majorana neutrinos: Strong enhancement by multiple soft scattering, JCAP 03 (2011) 042 [arXiv: 1012.3784] [INSPIRE].

[8] D. Besak and D. Bödeker, Thermal production of ultrarelativistic right-handed neutrinos: complete leading-order results, JCAP 03 (2012) 029 [arXiv:1202.1288] [INSPIRE].

[9] P.B. Arnold, G.D. Moore and L.G. Yaffe, Photon emission from ultrarelativistic plasmas, JHEP 11 (2001) 057 [hep-ph/0109064] [INSPIRE].

[10] P.B. Arnold, G.D. Moore and L.G. Yaffe, Photon emission from quark gluon plasma: complete leading order results, JHEP 12 (2001) 009 [hep-ph/0111107] [INSPIRE].

[11] P.B. Arnold, G.D. Moore and L.G. Yaffe, Photon and gluon emission in relativistic plasmas, JHEP 06 (2002) 030 [hep-ph/0204343] [INSPIRE].

[12] A. Salvio, P. Lodone and A. Strumia, Towards leptogenesis at NLO: the right-handed neutrino interaction rate, JHEP 08 (2011) 116 [arXiv:1106.2814] [INSPIRE].

[13] M. Laine and Y. Schröder, Thermal right-handed neutrino production rate in the non-relativistic regime, JHEP 02 (2012) 068 [arXiv:1112.1205] [INSPIRE].

[14] S. Caron-Huot, Asymptotics of thermal spectral functions, Phys. Rev. D 79 (2009) 125009 [arXiv: 0903.3958] [INSPIRE].

[15] M. Laine, Thermal 2-loop master spectral function at finite momentum, JHEP 05 (2013) 083 [arXiv: 1304.0202] [INSPIRE].

[16] B. Garbrecht, F. Glowna and M. Herranen, Right-handed neutrino production at finite temperature: radiative corrections, soft and collinear divergences, JHEP 04 (2013) 099 [arXiv: 1302.0743] [INSPIRE].

[17] T. Asaka, M. Laine and M. Shaposhnikov, On the hadronic contribution to sterile neutrino production, JHEP 06 (2006) 053 [hep-ph/0605209] [INSPIRE].

[18] M. Laine, Thermal right-handed neutrino self-energy in the non-relativistic regime, arXiv: 1209.2869 [INSPIRE].

[19] C.P. Kiessig, M. Plümacher and M.H. Thoma, Decay of a Yukawa fermion at finite temperature and applications to leptogenesis, Phys. Rev. D 82 (2010) 036007 [arXiv: 1003.3016] [INSPIRE].

[20] S. Caron-Huot, On supersymmetry at finite temperature, Phys. Rev. D 79 (2009) 125002 [arXiv:0808.0155] [INSPIRE].

[21] P. Aurenche, F. Gelis, G. Moore and H. Zaraket, Landau-Pomeranchuk-Migdal resummation for dilepton production, JHEP 12 (2002) 006 [hep-ph/0211036] [INSPIRE].

[22] B. Garbrecht, F. Glowna and P. Schwaller, Scattering rates for leptogenesis: damping of lepton flavour coherence and production of singlet neutrinos, arXiv:1303.5498 [INSPIRE]. 
[23] J.-S. Gagnon and M. Shaposhnikov, Baryon Asymmetry of the Universe without Boltzmann or Kadanoff-Baym equations, Phys. Rev. D 83 (2011) 065021 [arXiv:1012.1126] [InSPIRE].

[24] M. Laine, A. Vuorinen and Y. Zhu, Next-to-leading order thermal spectral functions in the perturbative domain, JHEP 09 (2011) 084 [arXiv:1108.1259] [INSPIRE].

[25] J. Ghiglieri, J. Hong, A. Kurkela, E. Lu, G.D. Moore and D. Teaney, Next-to-leading order thermal photon production in a weakly coupled quark-gluon plasma, JHEP 05 (2013) 010 [arXiv: 1302.5970] [INSPIRE].

[26] K. Kajantie, M. Laine, K. Rummukainen and M.E. Shaposhnikov, Generic rules for high temperature dimensional reduction and their application to the Standard Model, Nucl. Phys. B 458 (1996) 90 [hep-ph/9508379] [INSPIRE]. 